\title{
Two Stages in Visual Texture Segregation: A Lesion Study in the Cat
}

\author{
P. De Weerd, ${ }^{1}$ J. M. Sprague, ${ }^{2}$ E. Vandenbussche, ${ }^{1}$ and G. A. Orban ${ }^{1}$ \\ 'Laboratorium voor Neuro- en Psychofysiologie, Katholieke Universiteit te Leuven, Campus Gasthuisberg, Herestraat, B \\ 3000 Leuven, Belgium, and 2Department of Anatomy, Institute of Neurological Sciences, University of Pennsylvania, \\ School of Medicine, Philadelphia, Pennsylvania 19104-6058
}

\begin{abstract}
We have used five cats to investigate the effects of two distinct visual cortex lesions on the segregation of two different texture stimuli. The ablation of areas 17 and 18 (tier l) severely impaired the segregation between textures made of line elements differing in orientation, but spared the segregation between annulus and dot textures. In contrast, the ablation of those areas receiving direct afferents from areas 17 and 18 (tier II) destroyed the segregation for both texture stimuli. Strong deficits remained up to 1 year after the lesion, although limited recovery was observed after tier II lesions. We suggest that tier $I$ areas are involved in the local filtering of the texture elements, and that tier II areas compute texture differences on the basis of the filtered image provided by tier $I$ areas. The crucial contribution to texture segregation of visual areas belonging to a second level in the cortical hierarchy challenges the notion that texture segregation is entirely an early process in vision.
\end{abstract} tex]

IKey words: cat, texture, segregation, lesion, vision, cor-

A precondition for the perception of shapes and, ultimately, for the recognition of objects is the ability to distinguish surfaces on the basis of discontinuities in the retinal image. The border between different surfaces is often marked by coinciding changes in qualities such as luminance, color, motion, and also texture. Texture segregation refers to the capacity of the visual system to delineate surfaces on the basis of texture differences alone. In the present study, we investigated the neural substrate of texture segregation in the cat.

It is well established in humans that differences in texture provide a powerful cue for surface segregation (Julesz, 1981, 1986; Beck, 1982; Nothdurft, 1985). If texture elements of two surfaces differ in a single dimension such as orientation, brightness, size, or color, the border between these surfaces is especially conspicuous (Julesz, 1981; Julesz and Bergen, 1983; Bergen and Adelson, 1988). Texture segregation has also been

Received Dec. 16, 1992; revised June 21, 1993; accepted July 13,1993.
We are much indebted to C. Fransen for the training and testing of the animals
and to Drs. S. Raiguel and D. Xiao for the physiological recordings. The technical
support of G. Vanparrijs, G. Meulemans, P. Kayenbergh, and Y. Celis is kindly
acknowledged. For the histological preparations we thank W. Algeo. We are grate-
ful to A. Leuschow and A. Karni for critical reading of earlier versions of the
manuscript. P.D.W. held a junior research fellowship from the Research Council
for the University of Leuven. This work was supported by grants from the Uni-
versity of Leuven Research Council and from the National Research Council
(NFWO 3.0056.87) to G.A.O., and from the Research Foundation of the Uni-
versity of Pennsylvania to I.M.S., and NIH Grants EY-04906 and FY-02654.
a Correspondence should be addressed to G. A. Orban at the above address.
Copyright (C) 1994 Society for Neuroscience 0270-6474/94/140929-20\$05.00/0 demonstrated in monkeys (Schiller et al., 1990) and in cats (Wilkinson, 1986, 1990; De Weerd et al., 1992).

De Weerd et al. (1992) have shown that texture segregation in the cat varies with parameters such as element size and element spacing in a way similar to humans (Nothdurft, 1985; Nothdurft, 1990a). Furthermore, as in humans (Nothdurft, 1990a), the efficiency of texture segregation in the cat decreases as the positions of texture elements are randomized. In addition, in the cat, the discontinuity at the border between different textures is of prime importance during segregation, as reported in humans by Nothdurft (1985) and Landy and Bergen (1991). In light of the similarity of texture segregation in humans and cats, the cat is an interesting model in which to study the neural substrate of texture segregation.

In a previous study (Orban et al., 1990; Vandenbussche et al., 1991), we have used cats to determine the neural substrate of bar orientation discrimination. Bar orientation is not only a visual primitive of shape and pattern perception, it is also an elementary featurc of many texture patterns. Using texture patterns made of many small bars as a stimulus, we wished to identify the neural substrate of texture segregation and to $\mathrm{com}$ pare it with the neural substrate of bar orientation discrimination.

Vandenbussche et al. (1991) demonstrated a strong impairment in orientation discrimination of luminance-defined bars after a combined ablation of areas 17 and 18 (tier I areas). The deficit in the processing of a visual primitive such as bar orientation supports the notion that tier $\mathbf{I}$ areas are involved in early visual computations. Furthermore, the size of the ablation within tier I areas determined how much bar length, width, or contrast had to be reduced in order to observe a deficit. The relation between lesion size and filtering capacities supports the idea that tier I areas are involved in the local filtering of the retinal image. Significantly, the ablation of those areas to which tier I areas project directly (tier II areas) did not produce any of the above-described deficits (Vandenbussche et al., 1989). To the contrary, bar orientation discrimination remained intact even for the lowest contrasts and smallest bars tested. As a whole. these results are consistent with a modeling study of Devos and Orban (1990), in which it was shown that orientation of a single bar can be adequately represented without visual processing beyond an initial filtering stage.

In the present study, we used small bars as texture elements in a texture segregation task. The animals were required to detect a region of left oblique lines embedded in a background of right oblique lines, or vice versa. Successful performance in such a task requires a second processing step in addition to the initial filtering of the image. During that second stage (comparison 
stage), texturcs arc compared and texture differences detected. Further processing in the comparison stage includes the construction of boundaries and the extraction of shapes (e.g., Fogel and Sagi, 1989; Nothdurft, 1990b; Rubenstein and Sagi, 1990; Sagi, 1990). With our detection task, we only investigated the ability to distinguish between textures, and the terms "texture segregation" and "comparison stage" will be used solely in this restriclive meaning. The comparison stage could be implemented in tier I areas, but it is equally possible that this second stage is carried out in tier II areas, which receive strong input from tier I areas (Rosenquist, 1985). These two possibilities lead to different predictions, which have been tested in the present study.

In a computational framework (Brady and Grimson, 1981; Marr, 1982; Voorhees and Poggio 1988), texture segregation is typically considered an "early process" in vision. The finding that segregation occurs for brief stimulus exposures (Treisman and Gelade, 1980; Bergen and Julesz, 1981), and its effortless and preattentive nature are in agreement with that notion. Therefore, one might propose that texture segregation is achieved completely in tier I areas; that is, both the filtering and comparison stages might take place in tier I areas. In this view, the ablation of tier I areas would severely impair texture segregation, whereas the ablation of tier II areas would lcave tcxturc scgregation intact.

On the other hand, texture segregation may be achieved by tier II areas by computing differences between regions of a filtered image provided by tier I areas. The assumption that tier I areas only perform the initial filtering of the texture stimulus implies that deficits observed after a tier I ablation should depend on whether or not the texture stimulus matches the filtering capacities of the cat's remaining visual system. To test this line of thought, we introduced a second texture difference, defined by dots and annuli. The segregation of dot and annulus textures is less dependent on filtering constraints than textures made of line elements (see De Weerd et al., 1992), and therefore, one would expect the segregation between dot and annulus textures to be more resistant to an ablation of tier I areas. In contrast, assuming that the comparison operations presumably taking place in ticr II areas are common to all texture segregation tasks, a deficit after ablation of tier II areas should be equally strong for the two types of texture differences used.

\section{Materials and Methods}

\section{Subjects}

Five adult cats were used in the experiments: cats $57,59,63,64$, and 65. These animals participated in an earlier parametric study of texture segregation (De Weerd et al., 1992). They entered the present study 45 d preoperatively to record a baseline performance.

\section{Behavioral task and testing apparatus}

The cats were required to detect the left/right position of a square of a particular texture embedded in a different background texture. The detection trials were presented in an apparatus originally conceived by Berkley (1970). Our version of this apparatus consists of a box, with a Plexiglas chamber mounted on the front. The Plexiglas chamber's front panel is divided into two transparent response keys. Behind one key, the target texture surrounded by the background texture was presented; behind the other key, only the background texture was shown. After having viewed the stimuli through the response keys, the cats had to push one of these keys in order to make a response. A separator inserted between the two response keys prevented the animals from seeing the left and right stimuli simultaneously. The screen on which the stimuli were presented was positioned $25 \mathrm{~cm}$ in front of the response keys so that viewing distance from the screen was approximately $28.5 \mathrm{~cm}$. Food rewards (pureed beef) were given through an opening in the bottom of the Plexiglas front chamber, just below the response keys.

Each detection trial started with the presentation of a texture stimulus. A $0.35 \mathrm{sec}$ response delay period (RDP), during which responses wert ignored, was used to extinguish random responses immediately after stimulus onset. After expiration of the RDP, the animal was rewarded for pushing the response key behind which the target appeared (positive stimulus, or $\mathrm{S}+$ ). The $\mathrm{S}+$ was presented behind the left and right response keys in a balanced, pseudorandom order (see De Weerd et al., 1990b). All correct responses were rewarded, and incorrect ones were left unpunished. After the animal's response, the stimulus disappeared, and it had to wait for a $4 \mathrm{sec}$ intertrial interval (ITI) until the following stimulus was presented.

\section{Stimuli}

Stimulation apparatus. We presented the textures on a monochrome ATRIS screen. This screen has a $75 \mathrm{~Hz}$ refresh rate, and is characterized by a 18 by $36 \mathrm{~cm}$ surface containing 720 by 1440 pixels, respectively. Each individual pixel was either black $\left(0.08 \mathrm{~cd} / \mathrm{m}^{2}\right)$ or white $(24.02 \mathrm{~cd} /$ $\mathrm{m}^{2}$ ), which corresponds to a contrast between white and black of 2.48 $(\log [\Delta I / I])$. For technical reasons we only used the middle 1024 pixels of each 1440 pixel image line. Therefore, the displays shown in Figure 1 do not have a 2:1 aspect ratio as one might expect from the dimensions of the screen.

Types of texture stimuli. The standard detection target was an $8^{\circ}$ by $8^{\circ}$ square filled with one texture and surrounded by another. We used two different types of texture stimuli. In Figure $1, A$ and $B$, target and background texture are made of identical line elements and the target is defined solely by the $90^{\circ}$ orientation difference. Only the left and right oblique orientations were used to create the texture difference (line texture). Figure 1, $C$ and $D$, shows stimuli in which the target is defined by a square filled with annuli, surrounded by a background texture of dots (dot/annulus texture). To draw dots and annuli of matching area, we chose the inner diameter of the annuli equal to the diameter of the dots, and the outer diameter equal to $\vee 2$ times the inner diameter. In all stimuli used, texture elements were white on a black background.

We took special care to ensure there was no significant global luminance difference between target and background textures. Horizontal lines drawn on a CRT screen are slightly brighter than vertical lines, and the use of left and right oblique line elements (Fig. $1 A, B$ ) was the most straightforward way to circumvent that problem. Thus, in line texture stimuli, all elements were matched for luminance, length, and width (standard width was $0.2^{\circ}$ ). To equate the luminances of target and nontarget areas in dot/annulus textures, we had to overcome the fact that the specific properties of CRT screens cause dot elements to be slightly more luminous than annulus elements of equal area. In addition, inevitable rounding errors associated with the use of a digitized screen led to small differences in the number of pixels of which dots and annuli were composed, which could have led to perceptible luminance differences between target and background textures when using small texture elements. To solve these problems, we introduced a spccial calibration procedure in which the luminance of the annulus texture was matched to the dot texture. First, the dot texture was generated in an $8^{\circ}$ by $8^{\circ}$ window, and its global luminance measured using a Minolta luminance meter. Global luminances were obtained by averaging over large numbers of measurements taken at different locations in the texture. Then the corresponding annulus texture was generated in the same window, and its global luminance measured, after which we fine tuned the parameters used by the procedure that drew the annuli so as to add or leave out a few pixels at randomly chosen locations at the border of the annuli. After reaching the luminance match, the actual testing stimulus was drawn using the fine-tumed parameters, upon which luminances were remeasured. These steps were repeated for every single stimulus with which the animals were tested. The remaining global luminance differences increased as element size decreased, but within the range of sizes used during testing they remained smaller than $0.05 \mathrm{~cd} / \mathrm{m}^{2}$ and were imperceptible to us. It is worth mentioning that differences in luminance between individual dots and annuli were smaller than global luminance differences. The small differences in total area between dots and annuli, which resulted from our efforts to match their luminances, were equally imperceptible. Behavioral results obtained in this study show that the small luminance cue that remained in dot/annulus textures was too small to contribute to our results (see Fig. 17, and Discussion).

In both the dot/annulus and line textures, we varied element position randomization and spacing between elements. Element position ran- 
domization was manipulated by using two different texture conditions that are referred to as the regular and the randomized texture condition. In the regular texture condition, the texture elements were dispersed over the image following a predefined grid. As a consequence, the texture elements were organized in rows and columns (Fig. $1 A$ ). In the randomized texture condition, texture elements were displaced from the grid points by a distance equal to a maximum of $50 \%$ of the spacing between neighboring grid points, with the direction and distance of this displacement randomly distributed (Fig. $1 B, C, D$ ). The spacing between neighboring grid points is referred to as the element spacing. We used five element spacings of $4^{\circ}, 2^{\circ}, 1^{\circ}, 0.5^{\circ}$, or $0.31^{\circ}$. The densities corresponding to these five element spacings are $0.06,0.25,1,4$, and 10.56 elements per square visual degree, respectively. Element spacing was $4^{\circ}$ in Figure $1, A$ and $C$, and $2^{\circ}$ in $B$ and $D$.

When randomizing the positions of line elements, a procedure was used that minimized overlap (see De Weerd et al., 1992, for details). When randomizing the positions of dots and annuli, no overlap was allowed. For annulus diameters larger than $50 \%$ of the element spacing, the maximal displacement of the elements was reduced by the difference between half of the element spacing and the annulus diameter (Fig. $1 C$ ). The use of randomized textures eliminates consistent local luminance differences at the border between target and background textures.

During both training and testing procedures, the target was positioned randomly in the background texture. We also randomly exchanged figure and background textures. For the line texture, the target was filled with left oblique line segments and surrounded with right oblique elements, or vice versa. Dot/annulus textures could be made with a target of dots and a background of annuli, or vice versa. This procedure prevented the animals from solving the task by merely identifying the presence of a particular texture element in the positive stimulus. Rather, the animals were forced to use the texture difference as a cue to solve the segregation task.

\section{Behavioral procedures}

Since the efficiency of segregation is determined by the ratio between element size and element spacing rather than by absolute element size, we expressed length of the line elements relative to the element spacing, and this ratio is referred to as the relative length. Likewise, expressing the outer diameter of an annulus as a proportion of the element spacing results in a relative diameter. The sensitivity of our subjects towards a particular texture difference was assessed by measuring segregation performance as a function of relative element size at a constant element spacing. When line texture stimuli were used, relative length of the line elements was varied, keeping width at $0.2^{\circ}$. In dot/annulus texture stimuli, the relative diameter of the annuli was varied, keeping the area of annulus and dot elements equal to each other. Relative lengths and diameters were manipulated in a proportional way (multiplying or dividing a given value by a factor 1.1 ).

Training procedure. All five animals were trained with the differential exposure method (DEM; De Weerd et al., 1990a, 1992). For details about the preoperative training history, we refer to De Weerd et al. (1992). The same DEM was used to retrain the animals after the lesions. Briefly, a DEM session consists of 300 trials. In some trials, the negative stimulus disappears after a given period of time, whereas the positive (rewarded) stimulus remains in view until a response occurs. These cueing trials are mixed with trials in which the animals have to solve the task without assistance. Both the number of such cueing trials and the magnitude of the time cue are determined by the animal's performance. When the animal is able to distinguish the stimuli without cue (75\% correct in 20 trials), the stimulus difference is reduced, and the time cue reintroduced. This method has proven to be highly effective, as it decreased the time needed to reach the threshold in bar orientation discrimination by a factor of up to 5 , and an even larger gain was obtained using more difficult discriminations (De Weerd et al., 1990a). In the present study, the DEM was used to reduce the length of the line elements when using line texture stimuli, and in case of dot/annulus texture stimuli, the DEM was used to decrease the diameter of the dots and annuli.

Testing procedure. Detection thresholds were determined using a $73.5 \%$ correct Wetherill and Levitt (1965) staircase procedure. In this procedure, line length or annulus diameter (both of which are referred to as element size) was made dependent upon performance. Element size was reduced after three consecutive correct responses, or after two correct responses followed by an incorrect and a correct one. Element size was increased after an incorrect response, a correct response followed by an
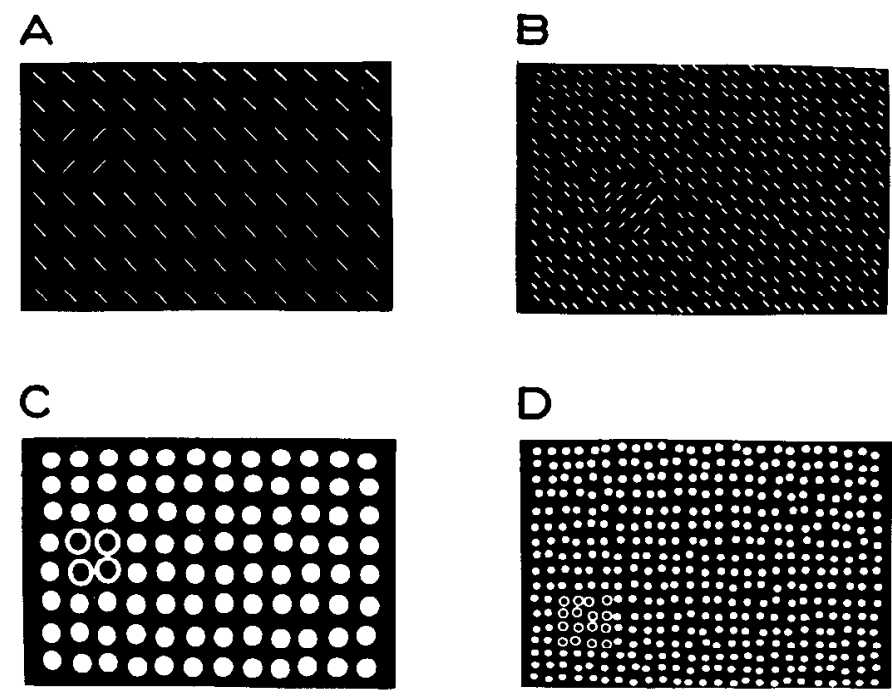

Figure 1. Representative examples of texture stimuli used in our experiments. $A$ and $B$ show line texture stimuli. In $A$, line elements are arranged in rows and columns (regular condition). In $B$, the position of the elements is randomized (randomized condition). $C$ and $D$ show dot/ annulus texture stimuli. The texture elements of the two dot/annulus texture stimuli are randomized in position (randomized condition). Large annuli and dots limit the degree of randomization $(C$; see Materials and Methods). Element spacing in $A$ and $C$ is $4^{\circ}$; in $B$ and $D$ it is $2^{\circ}$.

incorrect response, or after two correct responses followed by two incorrect ones. The staircases started at threshold level as determined from previous sessions. The first five trials of each staircase did not contribute to threshold estimation. Since the staircase measurements started at threshold, all reversal points were included in threshold calculation. Because of the proportional variation of element size, the threshold was calculated as a geometrical mean. In a single daily session, four staircase thresholds were determined, each staircase lasting 75 trials. We determined two thresholds in the regular texture condition and two in the randomized texture condition at a particular element spacing. The two staircase measurements in regular and randomized texture conditions were paired, but the order of the pairs was randomized between sessions.

In many cases, postoperative performance was so poor that the staircases yielded percentages of correct responses significantly lower than the $73.5 \%$ on which they were supposed to converge. Hence, in these instances, even those stimuli with the largest element sizes did not sufficiently control segregation behavior. To derive a criterion for stimulus control, we compiled the preoperative distribution of percentages correct per staircase, and used the mean minus 2 standard deviations as a cutoff point. In cat 65 , the preoperative average percentage correct was 73.7 , with a standard deviation of 4.4 (Fig. 2). Hence, the cutum point for stimulus control was $65 \%$ in this animal. In cats $57,59,63$, and 64 , the staircases converged on $75 \%, 77 \%, 76 \%$, and $72 \%$, and the cutoff points were put at $64 \%, 67 \%, 68 \%$, and $64 \%$, respectively. Measurements in which the percentage correct exceeded the cutoff point looked like valid staircases, showing a normal pattern of peaks and valleys for the majority of the trials, allowing us to calculate a threshold. As indicators of postoperative performance, we used the threshold values of those measurements in which stimulus control was reached, as well as the number of measurements meeting our criterion of stimulus control.

In a number of experiments, we also measured percentage correct responses while keeping the element size fixed at a relative length of 1.29 for line elements and a relative diameter of 0.95 for dots and annuli. We refer to these assessments as fixed stimulus measurements. Clearly, in conditions in which staircase measurements lack stimulus control, fixed stimulus measurements should also yield low percentages of correct responses.

Differences between experimental conditions, observed with either the staircase or the fixed stimulus method, were tested using a twotailed $t$ test $(t)$ for independent samples using separate variances, and by the Mann-Whitney $U$ test $(M)$. Parametric and nonparametric test 


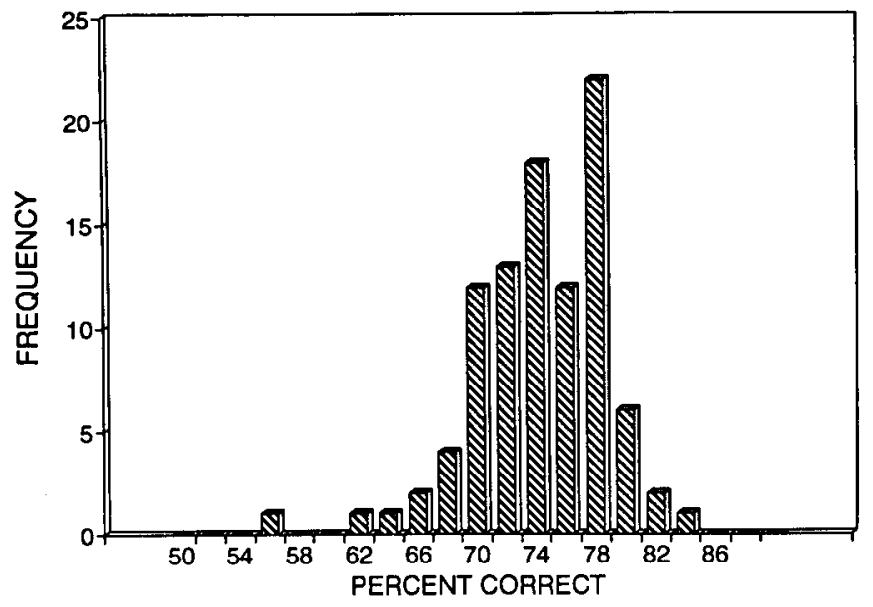

Figure 2. Preoperative distribution of the percentages of correct responses in each staircase for cat 65 . The average of this distribution was $73.73 \%$ and its standard deviation $4.46 \%$.

statistics yielded consistent results. Differences between conditions in the numbers of staircase measurements with stimulus control were tested using a Pearson $\chi^{2}$ statistic. In some experiments, completely randomized factorial ANOVAs (Kirk, 1968) were applied to analyze the data.

\section{Plan of the lesions}

The lesions were made using both anatomical and physiological criteria and were of two types. The first included areas 17 and 18, which share many physiological properties (Berkley and Sprague, 1979; Orban, 1984) and are the exclusive recipients of projections from laminae $\mathrm{A}$ and $\mathrm{A} 1$ of the dorsolateral geniculate nucleus dLGN. We refer to these areas as tier I.

A number of areas, including areas 19,20a, 21a, 21b, and two divisions of the lateral suprasylvian cortex (AMLS, PMLS; Palmer et al., 1978) receive direct projections from tier I areas (Rosenquist, 1985), thus constituting a secondary system of visual processing. These areas also receive thalamic afferents from dLGN laminae other than $\mathrm{A}$ and $\mathrm{Al}$, and from the lateral posterior pulvinar complex (Kosenquist, 1985). We refer to this group of areas as tier II, and this group was the target of our second type of lesion.

\section{Surgical procedures}

Anesthesia was induced by intramuscular injection of ketamine $(0.5 \mathrm{ml})$ followed by $1.0 \mathrm{ml}$ atropine. The femoral vein was cannulated, and Nembutal diluted 50:50 with saline was administered until respiration was stabilized at $10-16 / \mathrm{min}$ and no nociceptive reflexes could be evoked. Hair on top of the head was removed with a depilatory. The external meati were filled with an analgesic ointment, and the cat was placed in a stereotaxic instrument. Body temperature was maintained between $37^{\circ} \mathrm{C}$ and $37.5^{\circ} \mathrm{C}$. Surgery was carried out using rigorous, sterile techniques, at the end of which antibiotic (Kefzol, $200 \mathrm{mg}$ ) was given intramuscularly.

Cortical lesions were made by subpial aspiration of the gray matter, guided by the anatomical studies of Otsuka and IIassler (1962) and Sanides and Hoffmann (1969), and the physiological maps of Tusa et al. $(1978,1979,1981)$ and Palmer et al. (1978). The bone defects were covered with Gelfilm, the muscles were sutured in two layers, and the skin was closed with clips. The animals were awake and eating the next day, and could be returned to their home cages within $48 \mathrm{hr}$. Neurological tests (see Vandenbussche et al., 1991) were given weekly until responses were normal or until deficits were stable (2-3 weeks). By this time the incisions were healed and the animals were returned to the daily testing routine.

\section{Physiological and anatomical cvaluation of the lesions}

After postoperative training and testing were completed, the animals were prepared for single-unit recording using standard techniques (Orban et al., 1981a,b). Penetrations were made into the cortex surrounding the lesions (areas 18 and 19 in tier I animals, areas 17 and 18 in tier II animals) and the visual responsiveness of the units was assessed, as well as the receptive field locations. The locations of cells recorded in successful penetrations were marked with small electrolytic lesions for histological identification.

At the end of the physiological recording sessions (typically $2 \mathrm{~d}$ ), the anesthesia was deepened by injection of Nembutal and the animals were killed by intracardiac perfusion first with saline, then by $10 \%$ formalsaline. After hardening in the perfusate, the brains were blocked stereotaxically and removed from the skull. They were embedded in parlodion; coronal sections were cut at $40 \mu \mathrm{m}$ and stained alternately in cresyl violet for cell bodies and in Mahon for fibers. Representative stained sections were projected and drawn, and cortical areas were identified using anatomical criteria of Otsuka and Hassler (1962) and Sanides and Hoffmann (1969), and the physiological maps of Tusa et al. (1981). The cortical lesions were reconstructed, and the retinotopic position of retrograde atrophy in the dLGN was estimated using the study of Sanderson (1971). Lesion extent was plotted on the visual fields as determined in the studies of Tusa et al. $(1978,1979,1981)$ and Palmer et al. (1978). The visual field plots are useful in estimating the size and retinotopy of the lesions but do not show the cortical magnification. Therefore, the lesions were replotted on flat maps of the visual cortex, modified from Van Essen and Maunsell (1980). We added the approximate position of the different areas as derived from work of Tusa, Palmer, and Rosenquist cited above by putting in register the coronal levels defined in Figure 12 of Van Essen and Maunsell (1980) and in Figure 10 of Tusa et al. (1981) (Fig. 3). We added the approximate positions of the lines indicating visual field elevations and azimuths in areas 17, 18, and 19, derived from Figure 2 of Tusa et al. (1979).

\section{Results}

Anatomical results

\section{Tier I lesions}

Three cats $(57,63,65)$ had extensive bilateral lesions placed in areas 17 and 18. The extent of the lesion in cat 65 is shown in surface views (Fig. $3 A-D$ ) and in visual field plots of areas 17 , 18 , and 19 (Fig. $3 E-G$ ). These representations are the same as those we have used before (Orban et al., 1990; Vandenbussche et al., 1991; Flandrin et al., 1992). In addition, we have shown the extent of the lesions in cats 57,63 , and 65 on flattened maps of the visual cortex (Figs. 4-6). The lesions in areas 17 and 18 were large, and their extent was similar in all three animals. Despite occasional sparing of parts representing peripheral vision, all tier I lesions involved the representation of at least the central $10^{\circ}$ in both hemispheres. Small extensions of the lesion were found in the upper field representation of area 19 in cat 57 (Fig. 4). In cat 63, the lesion in area 19 included the entire representation of the upper field in the right hemisphere and parts of both upper and lower fields in the left (Fig. 5). In both hemispheres the representation of area centralis in area 19 was included in the lesion. In cat 65 , the representation of the lower peripheral field in area 19 was minimally involved in the right hemisphere, whereas a somewhat larger removal was present in the left hemisphere (Fig. 6).

The extent of the lesions was confirmed by the topography of retrograde degeneration in laminae $A$ and $A 1$ of the dLGN. In addition, there was considerable cell loss in lamina $\mathrm{C}$. It is noteworthy that physiological recordings revealed responsive neurors not only in area 19 (Figs. 4-6) but also in the spared parts of areas 17 and 18 in cal 57 (Fig. 4) and cal 63 (Fig. 5). There was good correlation between sites of responsive neurons and the parts of areas found to be spared in the anatomical reconstructions (see Figs. 3-6, 8, 9).

\section{Tier II lesions}

Two cats $(59,64)$ had extensive lesions in the complex of visual areas to which areas 17 and 18 project, and in area 7 (Figs. 79). Figure 7 shows the extent of the lesion in cat 59 in dorsal 


\section{CAT 65}
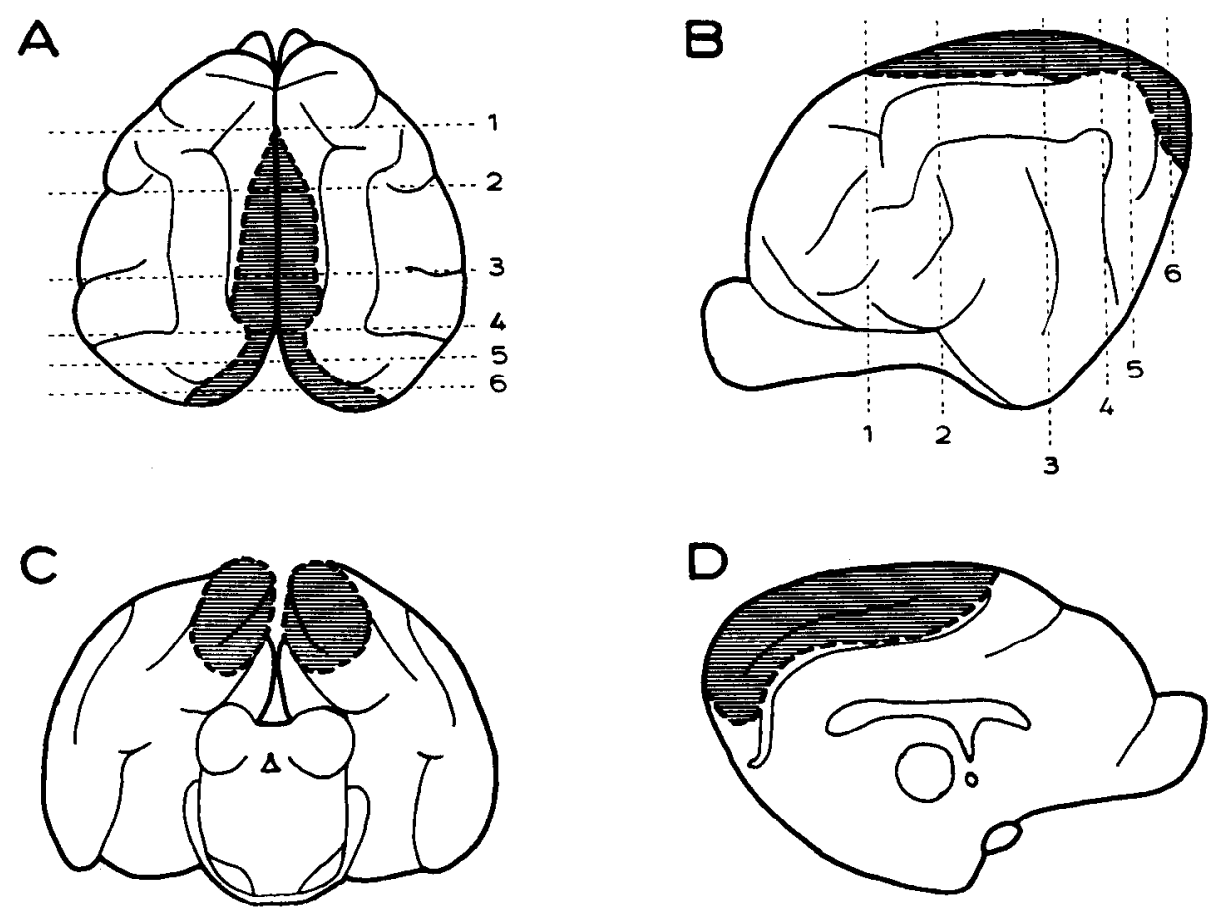

E AREA 17

F AREA 18

G AREA 19
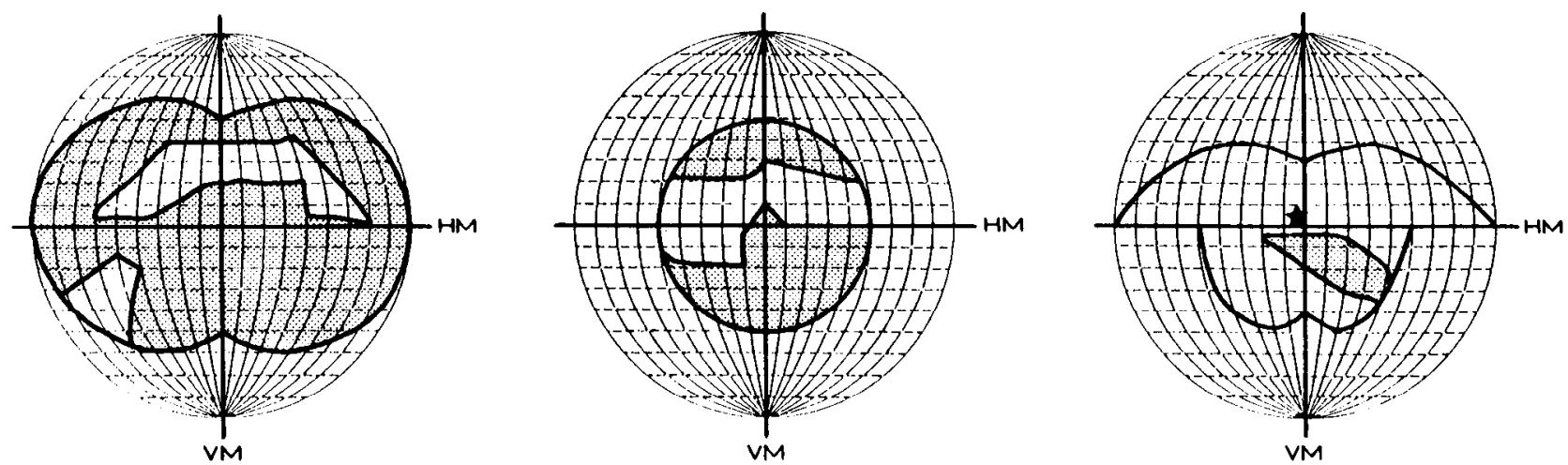

Figure 3. Surface views of the tier I lesion in cat 65 (A, dorsal; $B$, lateral; $C$, posterior; and $D$, medial). Gray areas show the extent of removed cortex. Horizontal dotted lines in $A$ and vertical lines in $B$ indicate the levels of coronal sections selected for detailed reconstruction. The splenial sulcus is opened to show the extent of the lesion in the superior bank. Lesion extent in areas 17, 18, and 19 was also plotted in visual field coordinates $(E-G)$. Gray zones indicate lesion extent and lighter zones show spared tissue. Azimuths and elevations are indicated in steps of $10^{\circ}$. The star indicates the receptive field positions of three area 19 cells found to he visually driven in the recording session hefore death. $H M$, horizontal meridian; $V M$, vertical meridian.

and lateral surface views of the brain. In both animals, areas 7 and 21 a were completely removed (Figs. 8,9 ). In addition, most of area $20 \mathrm{a}$ and most of PMLS, including their representation of the area centralis, was removed (Figs. 8, 9). AMLS was spared bilaterally in both animals. Area 21 b was largely removed, except for a part of the area centralis representation lying deep in the posterior suprasylvian sulcus; $21 \mathrm{~b}$ was wholly spared in the right hemisphere of cat 64 (Fig. 9A). The entire upper field representation of area 19 was removed in both animals; this lesion extended to $-5^{\circ}$ in cat 59 (Fig. 8), and to $-10^{\circ}$ in the lower field of cat 64 (Fig. 9). Much of the lower field representation that lies in the lateral gyrus was intentionally spared to avoid possible undercutting of the white matter innervating areas 17 and 18 . Examination of the dLGN revealed no degeneration in laminae $\mathrm{A}$ and $\mathrm{Al}$, and we conclude that areas 17 and 18 were functionally intact in both animals. This was con- 


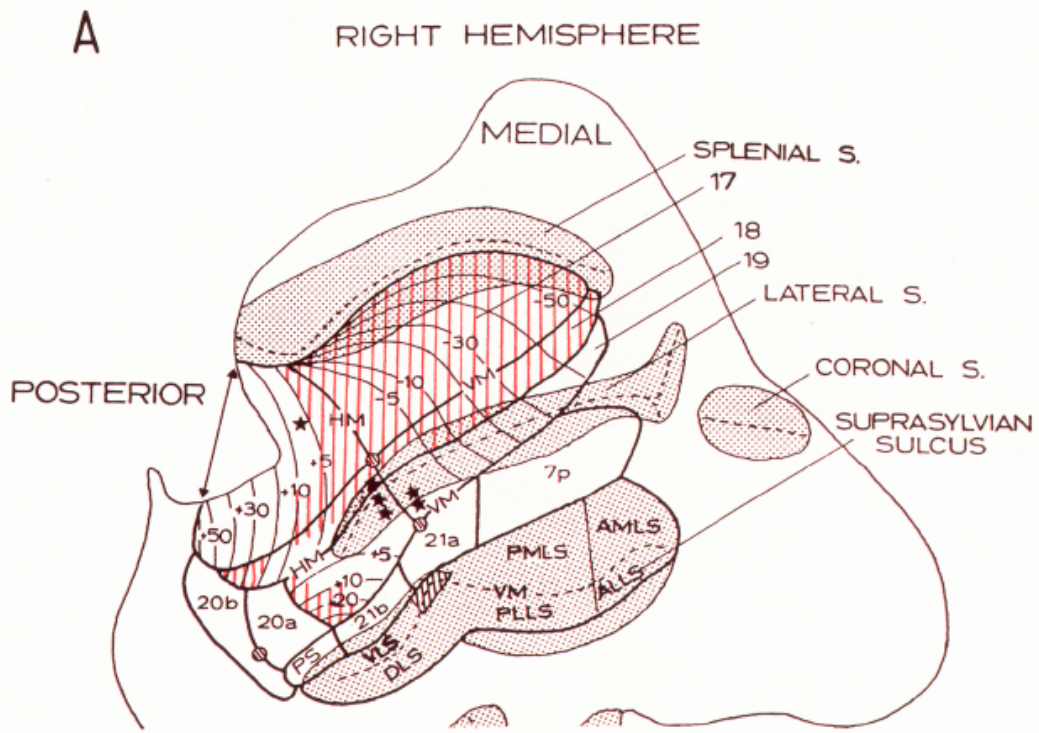

Figure 4. Flat map of the tier I lesion in cat 57 in right $(A)$ and left $(B)$ hemisphere. Stippled areas indicate sulci, after opening. The fundus of each sulcus is indicated by a dashed line. The borders of different areas are indicated by a heavy line. The red hatched area represents the extent of the lesion. The small, circular or elliptic, black hatched regions represent a part of cortex representing central vision. Elevation lines are shown and labeled in $A$. Labeled azimuth lines and unlabeled elevation lines are shown in $B$. The stars in $A$ represent the anatomical positions of 12 visually driven area 19 cells, 4 area 18 cells, and 3 area 17 cells recorded in two penetrations. Several penetrations were made into the left hemisphere $(B)$, all of them in damaged parts of area 18 , which explains the absence of visually driven cells. $H M$, horizontal meridian; $V M$, vertical meridian; $A M L S$, anterior medial lateral suprasylvian area; $A L L S$, anterior lateral lateral suprasylvian area; $P M L S$, posterior medial lateral suprasylvian area; $P L L S$, posterior lateral lateral suprasylvian area; $V L S$, ventral lateral suprasylvian area; $D L S$, dorsal lateral suprasylvian area; $P S$, posterior suprasylvian area.

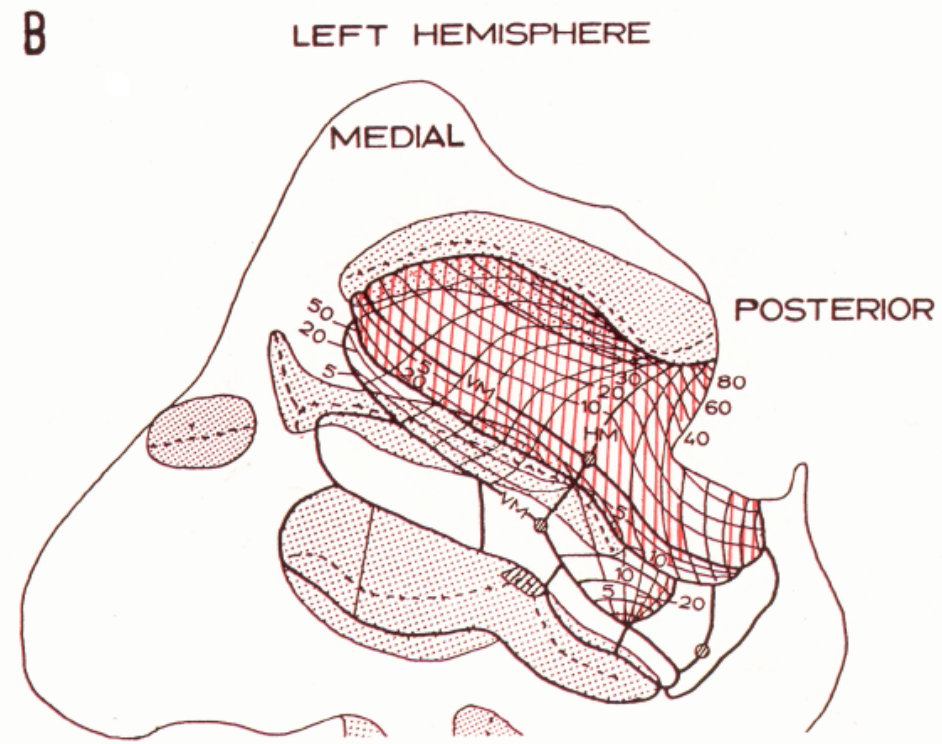

firmed by physiological recordings done just before death. The positions of the responsive neurons are indicated in Figures 8 (cat 59) and 9 (cat 64).

\section{Behavioral results}

\section{Strategy of behavioral testing}

We divided the behavioral results into four observation periods. In the first, preoperative period, we used optimal texture parameters (De Weerd et al., 1992) to establish a preoperative baseline. The standard element spacings used in period 1 for line textures and dot/annulus textures were $2^{\circ}$ and $1^{\circ}$, respectively. Standard target size was $8^{\circ}$ by $8^{\circ}$. During the second observation period, extending for 3 months postoperatively, we assessed the immediate effect of the lesion, using the same standard stimulus settings. In a third period, which extended up to 8 months after surgery, we carried out a number of parameter variations. Using the line texture, we studied the effects of spacing, line width, and target size. In addition, the effect of adding a luminance cue to the orientation difference was investigated. For the dot/annulus texture, we investigated the effect of element spacing. In the case of line textures, period 3 ended with some additional measurements at the standard element spacing. In a final, fourth period, which lasted up to 18 months after surgery, we retrained and retested the animals at different element spacings. The data obtained in the four different observation periods are plotted in Figures 11-14, in which observation periods are labeled 1-4. The results of parametric variations are shown in Figures 15-17. Before presenting the results in detail, we present an overview of the results in Figure 10.

\section{Overview of results at standard stimulus settings}

Figure 10 shows results obtained in standard conditions immediately after the lesion (observation period 2), results in the standard condition obtained at the end of period 3 (if available), and the result of retraining (period 4). It also offers a comparison with preoperative performance (period 1). The data were pooled 


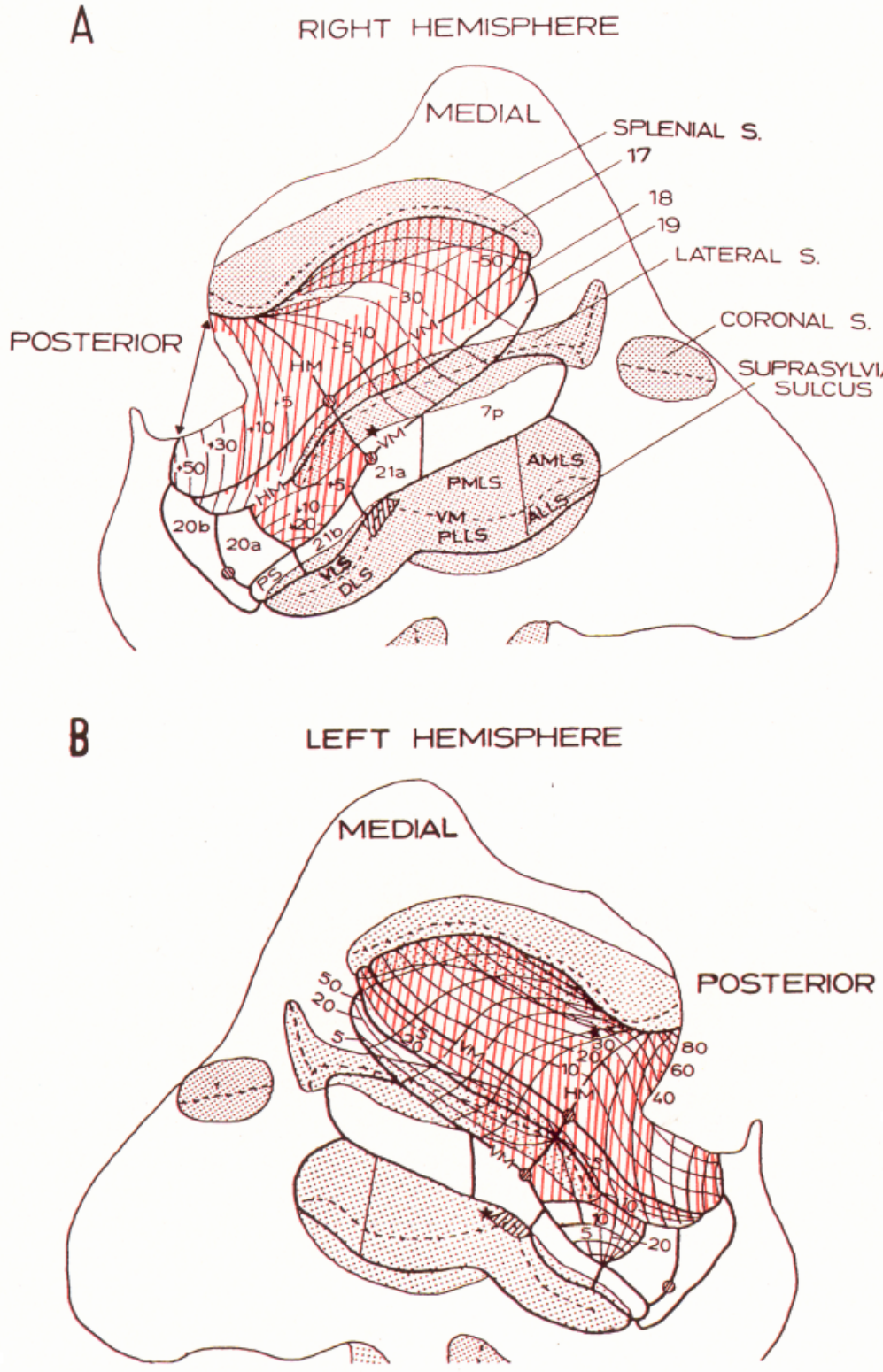

Figure 5. Flat map of the extent of the tier I lesion in cat 63. Stars in $A$ and $B$ represent the anatomical position of visually driven neurons in area $19(A)$, PMLS $(B)$, and area $17(B)$. Conventions are as in Figure 4. over cats, resulting in four summaries, one for each combination of texture stimulus and lesion type. The upper part of each summary shows the percentage of cases in which staircase measurements yielded stimulus control, and the lower part shows the average magnitude of those threshold measurements meeting the criterion for stimulus control.

Figure 10 illustrates the three main findings of this study. First, during observation period 2, tier I lesions caused a severe deficit in the segregation of line textures but left segregation of dot/annulus textures largely intact (Fig. 10A,B). The strong deficit in line texture segregation after tier I lesions was evident in the sharp drop in the number of staircases with stimulus control as well as in the increase in threshold size. While tier I ablations essentially spared the segregation of dot/annulus textures, tier II ablations indifferently affected the segregation of all texture types used (Fig. 10C,D). Second, Figure 10 shows that the immediate postoperative deficit (period 2) was much more severe after tier II lesions than after tier I lesions. After tier II lesions, stimulus control was reached in only $7 \%$ of the staircase measurements; after tier 1 lesions this was $64 \%$ (pooled over cats, regular/randomized conditions, and texture stimulus types). Third, and somewhat paradoxically, although tier II lesions caused more pronounced immediate deficits than tier I lesions, recovery after tier II lesions was modest but clear, in contrast to the virtually stable performance after tier I lesions. The recovery after tier II lesions was clear both in the number of staircases reaching stimulus control and in the size of the resulting thresholds. In the following sections, we chronologically present the detailed results obtained in the different postoperative observation periods.

\section{Description of immediate postoperative deficits: tier I lesions}

In this section, we compare performance before (observation period 1) with performance after (observation period 2) removal of areas 17 and 18. We first describe the results obtained with the line texture stimulus, followed by a report of the deficits 

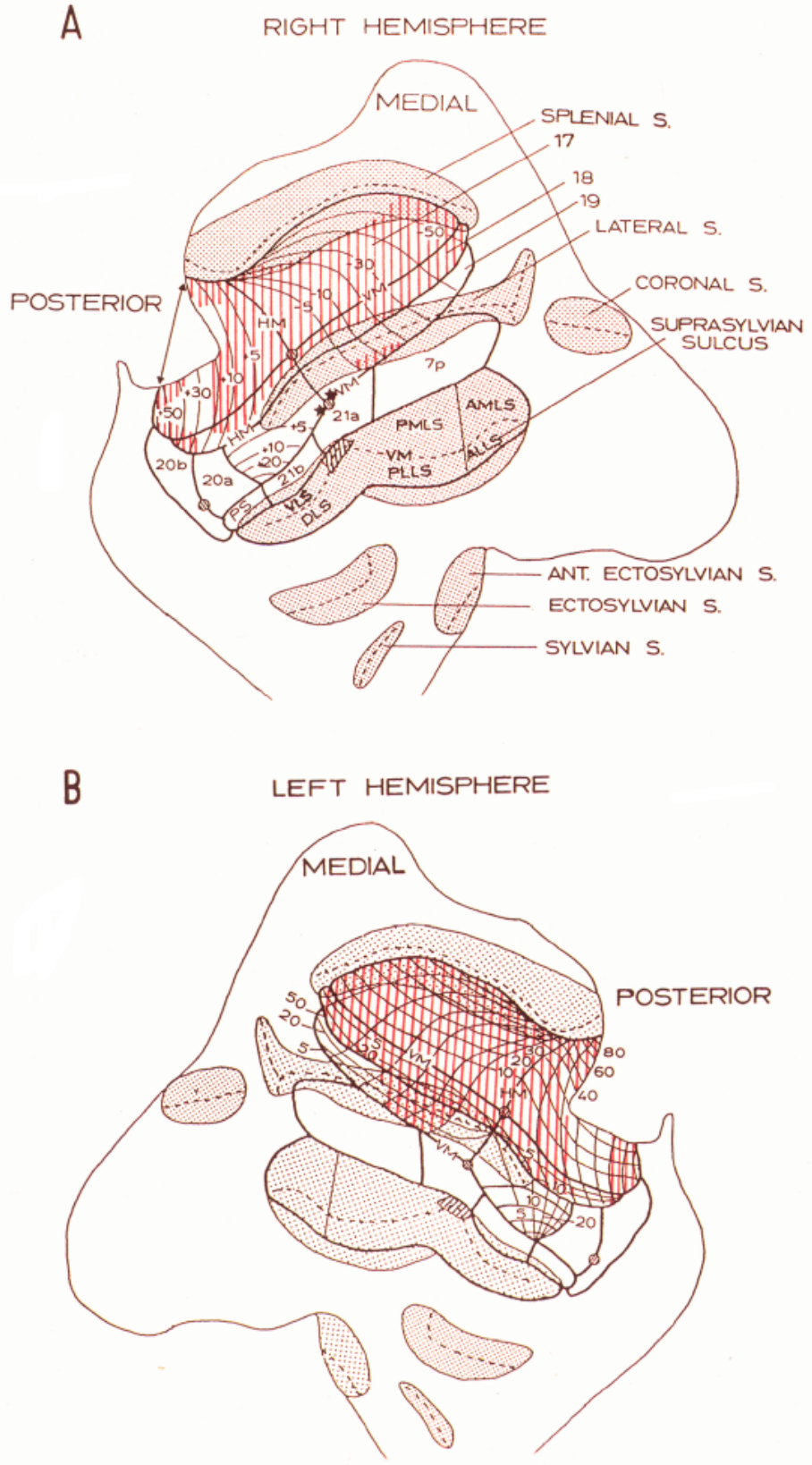

Figure 6. Flat map of the extent of the tier I lesion in cat 65. Stars in $A$ indicate the anatomical position of single units that could be visually driven in area 19 (see also Fig. 3). Conventions are as in Figure 4.

observed using dot/annulus textures. For both stimulus types, we first present our findings in the regular texture condition, followed by the results in the randomized condition. Average thresholds were calculated on staircases in which the criterion for stimulus control was reached.

With regular line textures (Fig. 11), cats 57,63, and 65 reached preoperative thresholds of a similar magnitude, the average being 0.53 . After the tier I ablation, average relative length thresholds rose to 1.17 in cat 57 and 1.20 in cat 65 . Cat 63 never reached stimulus control, and also cat 57 reached stimulus control in less than half of the staircase measurements. For randomized line textures (Fig. 11), the preoperative thresholds reached a value of 0.66 (averaged over cats), which is a little

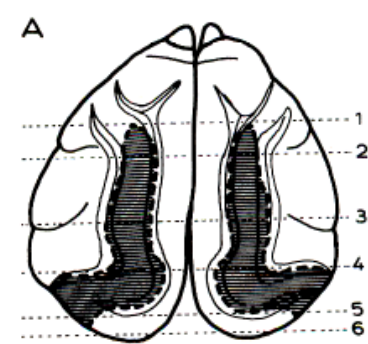

CAT 59

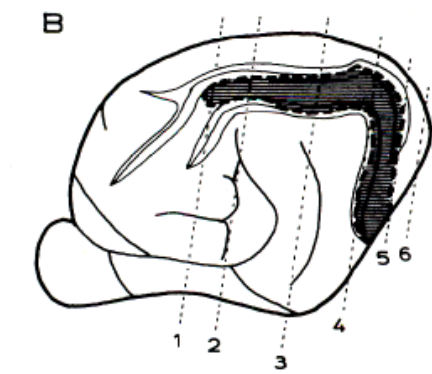

Figure 7. Surface views of the brain in cat 59 after a tier II lesion (dorsal in $A$, lateral in $B$ ). Conventions are as in Figure 3. The lateral (marginal) and suprasylvian sulci are opened to show the extent of the lesion into the upper and lower banks.

higher than in the regular condition. Postoperatively, the segregation of randomized line textures was heavily impaired, more so than the segregation of regular line textures. The animals systematically failed to reach stimulus control in the randomized condition, with only a few exceptions in cats 57 and 65 (Fig. 11). Hence, our results suggest that a difference remained between regular and randomized texture conditions after the ablation of tier I areas. The less efficient segregation in the randomized condition might be related to the elimination of local luminance cues at the border between textures (see De Weerd et al., 1992), or to the addition of luminance noise to figure and background textures (Rubenstein and Sagi, 1990).

It is worth mentioning that fixed stimulus measurements, during which the percentage correct was measured for a maximal element size (see Materials and Methods), confirmed the poor nature of residual segregation performance. In cats 57,63 , and 65 we found percentages correct of 57,52 , and 59 in the randomized condition, and slightly higher percentages of 67,59 , and 75 in the regular condition. The difference between regular and randomized conditions was significant in all cats $(M=275$, $p<0.001$ in cat $57 ; M=116, p<0.011$ in cat $63 ; M=144$, $p<0.001$ in cat 65 ).

Contrary to the texture composed of lines, segregation of the dot/annulus textures was much less affected by tier I lesions (Fig. 12). The cats had no trouble achieving stimulus control and reached surprisingly small thresholds. In the regular condition, preoperative relative diameter thresholds were 0.53 and 0.47 in cats 57 and 65 , respectively. After surgery, thresholds were elevated to average values of 0.78 and 0.68 , again in cats 57 and 65 , respectively. Although relatively small, this increase was significant in both cats $(t=-8.00, \mathrm{df}=29, p<0.001$ in cat $57 ; t=-12.86, \mathrm{df}=64, p<0.001$ in cat 65 ). Using randomized dot/annulus textures, average relative diameter thresholds increased from 0.69 preoperatively till 0.80 in cat 57 , and from 0.64 to 0.72 in cat 65 . Again, these increases were small but statistically significant $(t=-3.92, \mathrm{df}=33, p<0.001$ in cat $57 ; t=-4.27$, df $=53, p<0.001$ in cat 65 ). As observed for line textures, there was a small difference in segregation performance observed in regular and randomized conditions $(t$ $=-3.096, \mathrm{df}=27, p<0.005$ in cat $57 ; t=-5.219$, df $=83$, $p<0.001$ in cat 65 ).

\section{Description of immediate postoperative deficits: tier II lesions}

Whereas tier I lesions preferentially affected segregation of line textures, and left the segregation of dot/annulus textures par- 


\section{CAT 59}

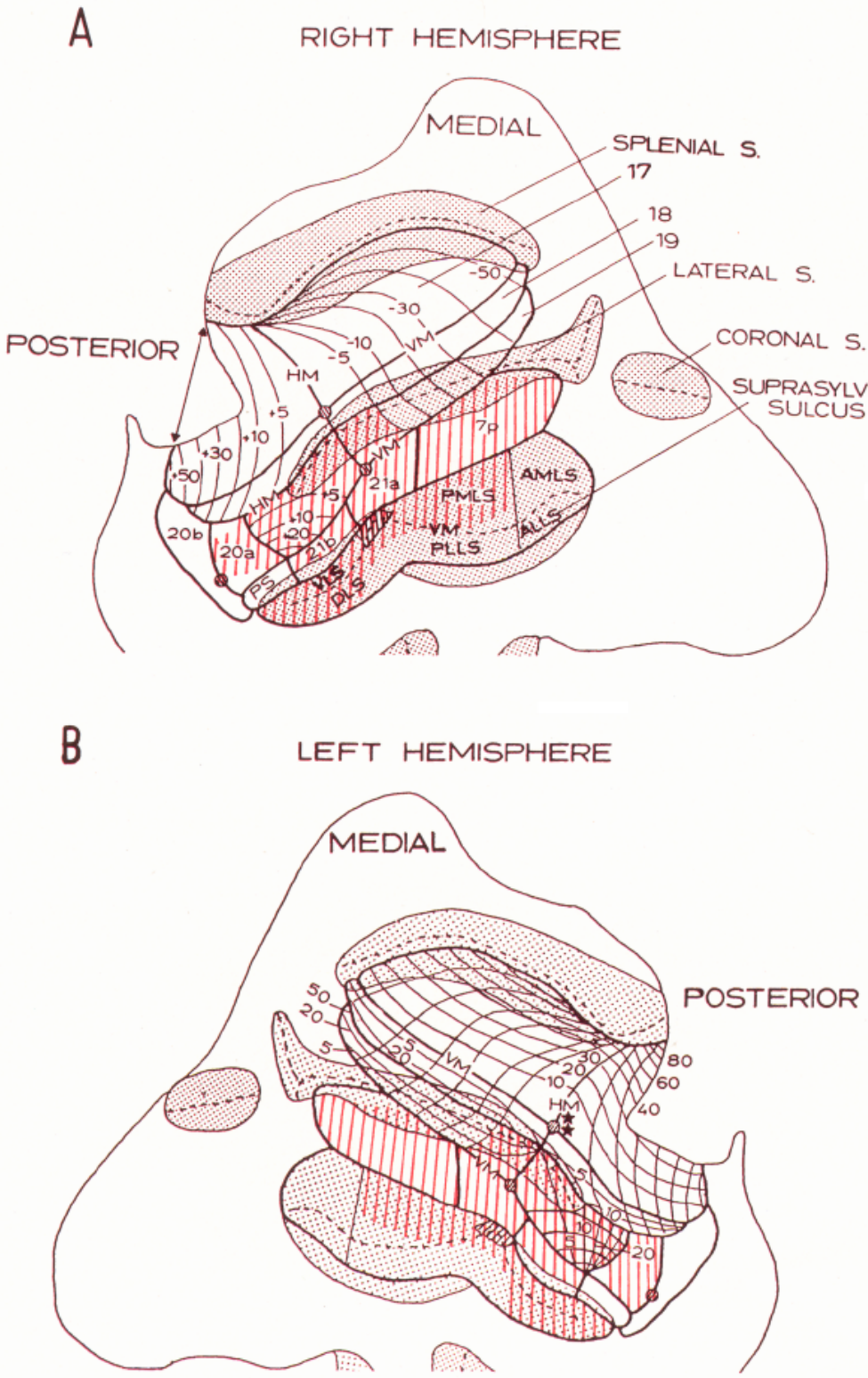

Figure 8. Flat map of the extent of the tier II lesion in cat 59. Stars in $B$ show the positions of nine cells recorded in a single penetration in area 17 . Conventions are as in Figure 4.

tially intact, tier II lesions caused a clear-cut deficit in the segregation of both classes of texture stimuli. The results are presented for the line texture first, and then for the dot/annulus texture.

In both cats 59 and 64, the deficit caused by the tier II ablation was similar for regular and randomized line textures (Fig. 13). In the large majority of sessions there was no stimulus control of segregation behavior in either condition. Fixed stimulus measurements revealed average percentages correct of 66 and 59 in the regular condition, and lower percentages of 58 and 55 in the randomized condition, in cats 59 and 64, respectively. This difference between regular and randomized conditions was significant $(M=164, p<0.002$ in cat $59 ; M=183, p<0.04$ in cat 64$)$.

For the dot/annulus texture, we obtained data from cat 64 only. Again, the effect of the tier II lesion was devastating (Fig.
14). In none of the staircase testing sessions could we obtain stimulus control, neither in the regular nor in the randomized condition. The fixed stimulus method yielded extremely low values: average percentages correct were as low as 54 and 58 in regular and randomized conditions, respectively. Hence, in contrast to the effect of tier I lesions (Fig. 12), the segregation of $\mathrm{dot} /$ annulus textures was heavily impaired after removal of tier II areas.

\section{Exploration of lesion deficits at other parameter settings}

The lesion effects presented in previous sections were observed at stimulus settings that were optimal in intact animals, but not necessarily in animals with lesions. Therefore, we assessed the lesion deficits for a variety of other stimulus settings. Using the line texture, we did a parametric study involving variations of spacing, line width, element position randomization, and target 


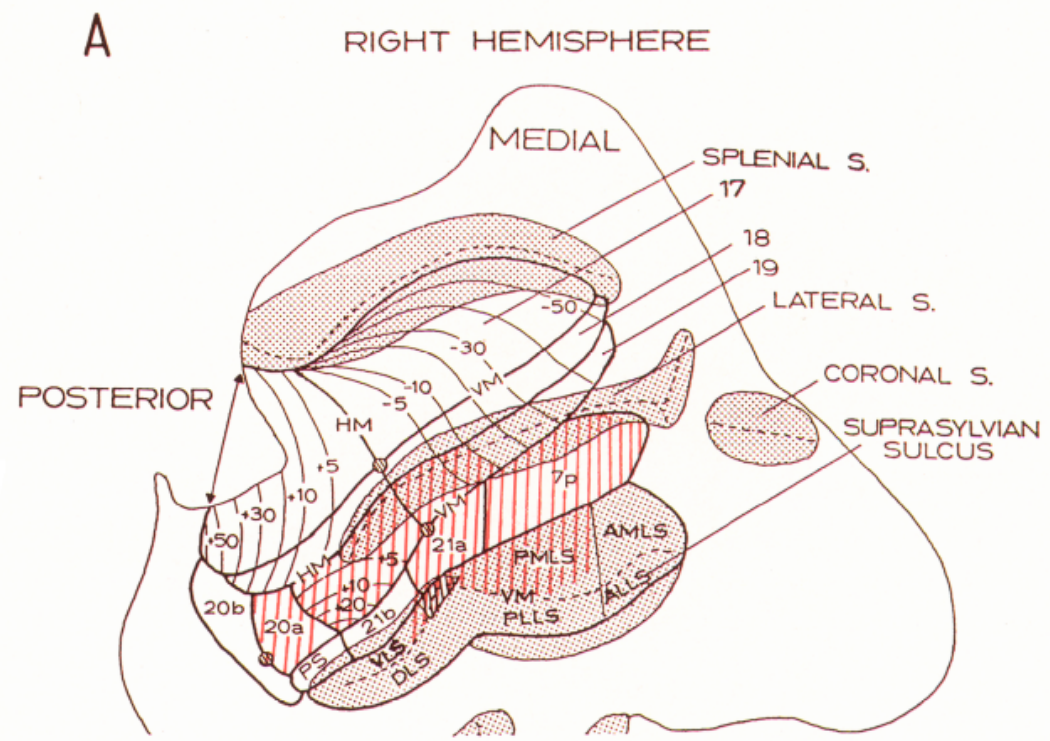

Figure 9. Flat map of the extent of the tier II lesion in cat 64. Stars in $B$ indicate 11 cells positioned near the border between areas 17 and 18 , recorded in two penetrations. Conventions are as in Figure 4.

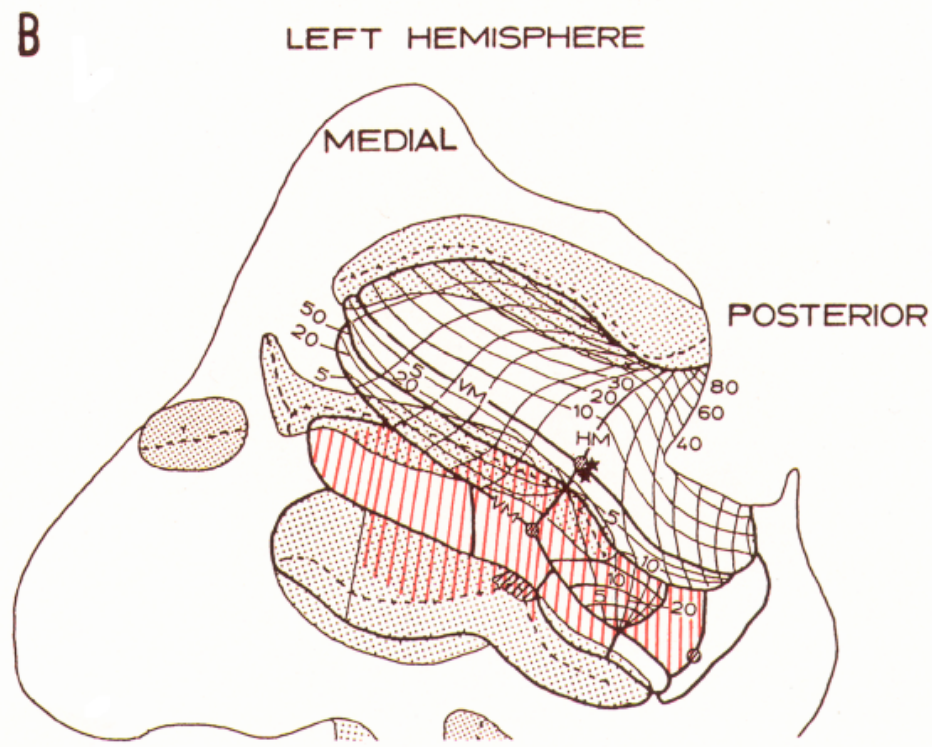

size. Using the dot/annulus texture, we manipulated spacing and element randomization. All these experiments were carried out during observation period 3 .

Preoperatively, spacing was the most important determinant of line texture segregation, a $2^{\circ}$ spacing allowing efficient segregation $(73.5 \%$ correct) of line textures containing elements only half as long as the element spacing, whereas relative lengths required for a $73.5 \%$ correct performance were significantly larger than 0.5 at $1^{\circ}$ and $4^{\circ}$ spacings. Changes in width, and element position randomization had a small though statistically significant effect (De Weerd et al., 1992). The result of the manipulation of spacing, element position randomization, and width after tier I lesions (fixed stimulus measurements, see Materials and Methods) is shown on the left in Figure 15; data obtained after the tier II lesions are shown on the right. ANOVA of the data in the individual cats showed that the postoperative effects of spacing, element position randomization, and also width in a few cases, were statistically significant. In all five cats, the most important experimental factor after the lesion was element position randomization, which accounted for $34 \%$ of the variance, compared to $16 \%$ preoperatively. Spacing determined an average of only $8 \%$ of the experimental variance after tier I lesions compared to $29 \%$ after tier II lesions, and $35 \%$ before the lesions. This fits with the clearer optimum at the $2^{\circ}$ spacing seen after tier II lesions. Note also that segregation at the $4^{\circ}$ spacing, with line elements as long as $5.6^{\circ}$, remained poor. Figure 15 clearly illustrates that stimulus settings other than a $2^{\circ}$ spacing and a $0.2^{\circ}$ line width did not produce any clear advantage. Furthermore, changes in target size both at a $2^{\circ}$ and a $1^{\circ}$ spacing did not significantly influence the segregation of line textures. Hence, the standard settings used during immediate postoperative testing were accurate choices to evaluate the lesion deficits.

Using the dot/annulus texture, we manipulated spacing in cat 65 (tier I lesion) and in cat 64 (tier II lesion) (Fig. 16). Segregation of dot/annulus textures after the tier I lesion was only slightly affected at the standard $1^{\circ}$ spacing. The deficit also showed up 


\author{
LINE-TEXTURE \\ STIMULUS
}

\section{DOT/ANNULUS-IEXTURE STIMULLUS}

TIER I LESION
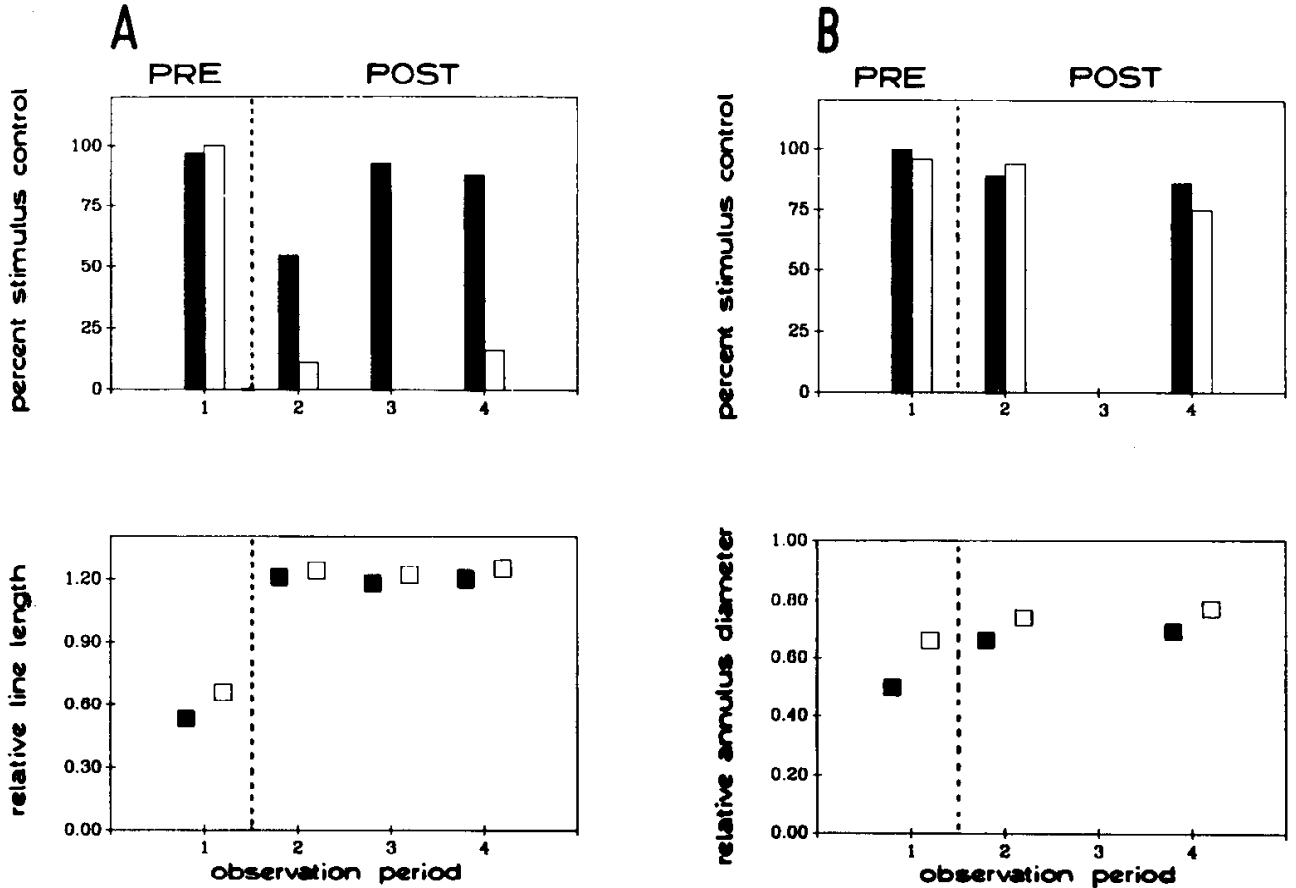

\section{TIER I LESION}

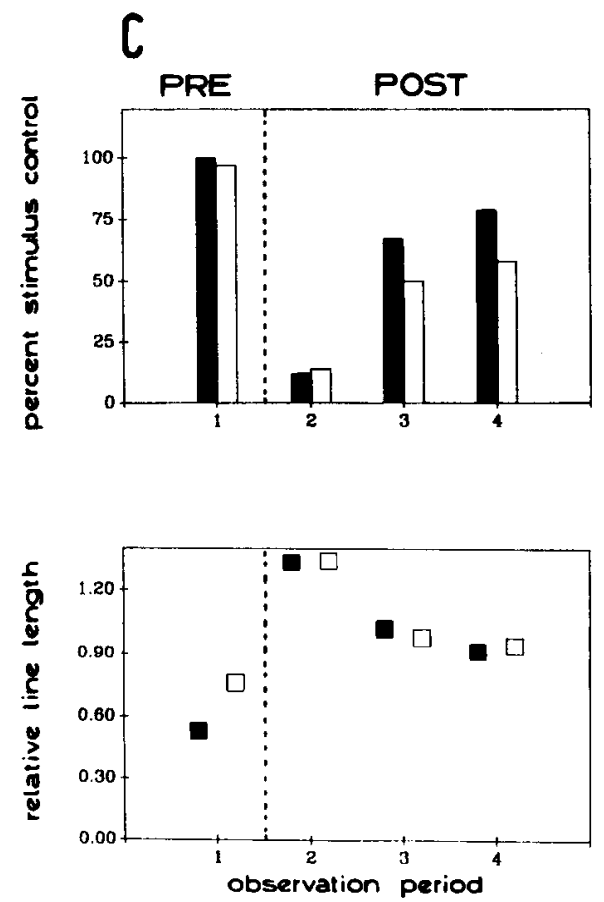

B

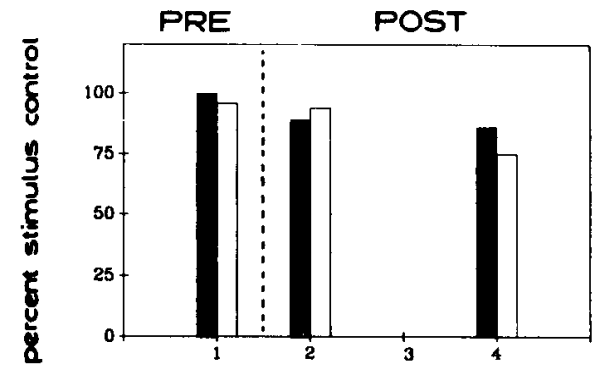

Figure 10. Summary of segregation performance for each combination of texture stimulus type and lesion type, after pooling of the data over cats. $A$ (cats 57,63, and 65) and $B$ (cats 57 and 65) show results obtained after tier I lesions with line texture and dot/annulus texture, respectively. $C$ (cats 59 and 64 ) and $D$ (cat 64 ) present results for line texture and dot/annulus texture observed after tier II lesions. For each of the four combinations, the upper graph shows the number of thresholds meeting the criterion for stimulus control, expressed as a percentage of the total number of observations (percent stimulus control), for observation periods $1-4$ (data pooled over cats). The lower graph in each combination shows the average of the thresholds meeting the criterion of stimulus control obtained in periods $1-4$. Period 1 shows preoperative baseline performance. $\mathrm{Pe}$ riod 2 shows measurements taken in the first 3 months after surgery. With line texture stimuli, we assessed spontaneous recovery during period 3 , which extended up to 8 months postoperatively. Period 4 shows results obtained after retraining. The vertical dashed line separates pre- from postoperative periods, labeled $P R E$ and $P O S T$. Solid bars and symbols refer to the regular texture condition; open bars and symbols, to the randomized texture condition. No data were gathered for period 3 in $B$ and $D$. N.S. in $D$ indicates that no stimulus control was reached and no thresholds could be determined. at other spacings and was statistically significant $[F(1,48)=$ $37.82 ; p<0.001$ ] (Fig. 16). The deficit was most pronounced at the smallest $\left(0.31^{\circ}\right)$ and at the largest spacings $\left(4^{\circ}\right)$. At the $4^{\circ}$ spacing (in which the animal was presented with elements up to $4^{\circ}$ in diameter), we were unable to obtain stimulus control.
Hence, in this case, a substantially larger deficit was observed by moving away from the optimal spacings of $1^{\circ}$ and $0.5^{\circ}$. However, Figure 16 also shows that pre- and postoperative curves were qualitatively similar, showing optimization of performance at intermediate spacings. Note also the significant effect of el- 


\section{A REGULAR}

\author{
B
}

\section{RANDOM}

\section{CAT 57}
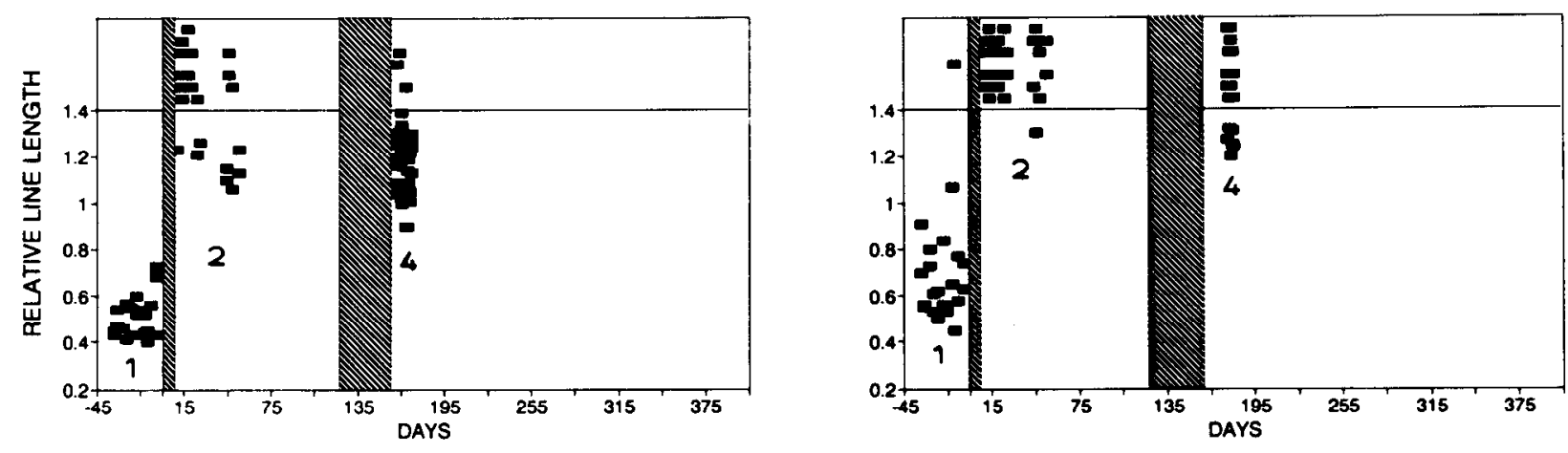

CAT 63
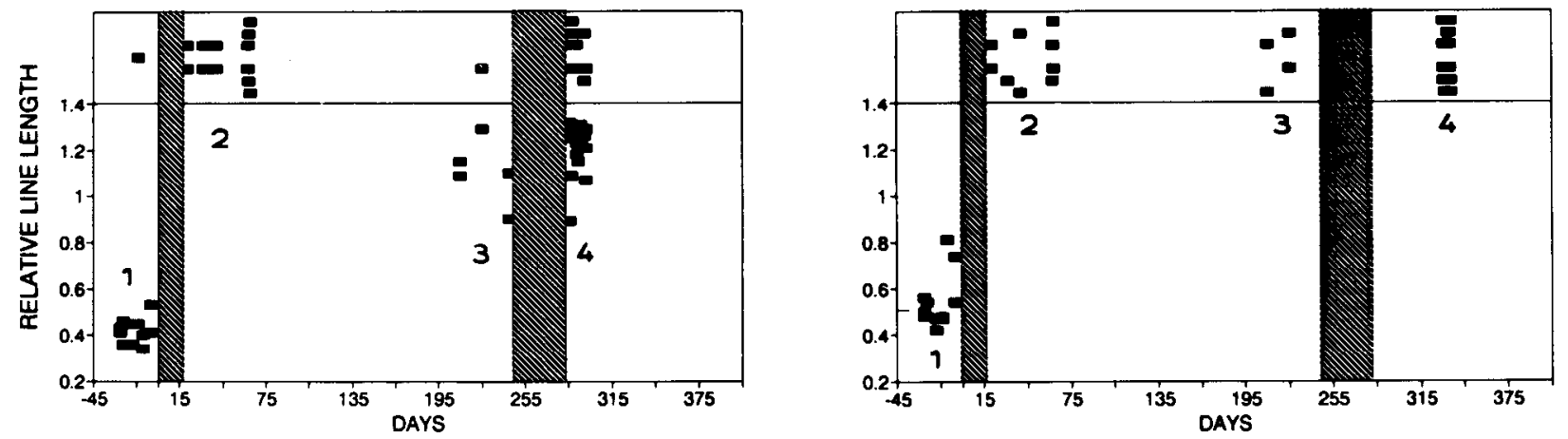

CAT 65
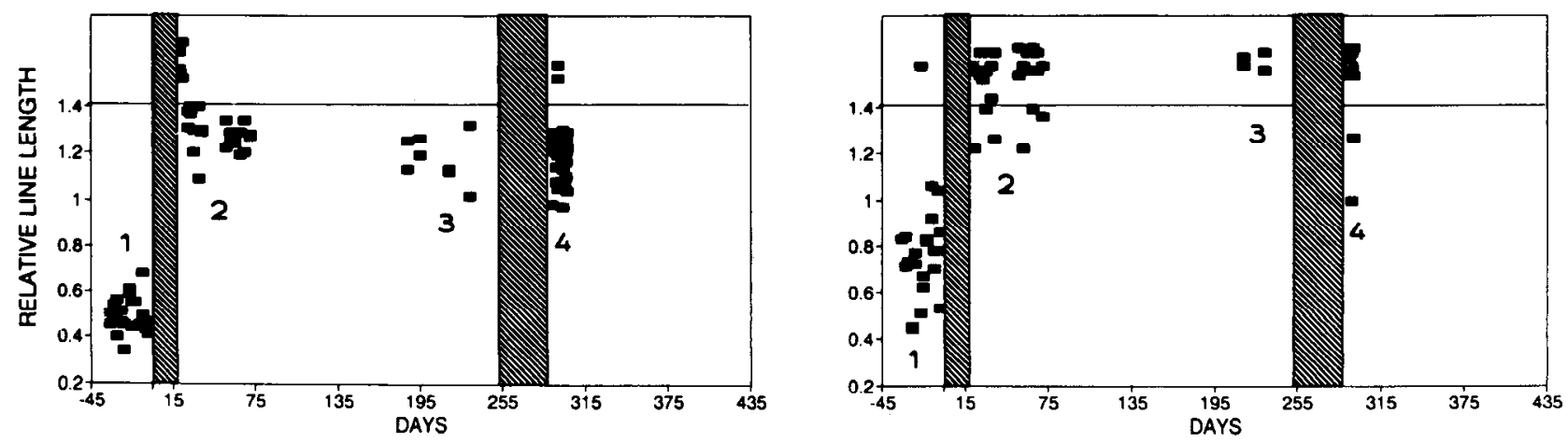

Figure 11. Line texture segregation after a tier I lesion. Segregation performance of cats 57,63 , and 65 is plotted as a function of time before (negative numbers, period 1) and after (positive numbers, periods 2-4) the tier I lesion. Results obtained with the regular line texture are shown on the left; those obtained with the randomized texture are shown on the right. Element spacing was $2^{\circ}$. Segregation was assessed with the staircase procedure. The threshold measurements meeting our criterion of stimulus control (see Materials and Methods) are shown below the 1.4 relative length boundary (indicated by the horizontal line). Measurements showing absence of stimulus control were plotted above this boundary, and their ordinal values were chosen arbitrarily in order to separate symbols. The left cluster of values labeled $l$ (period 1 ) represents preoperative values, followed by surgery and a recovery period indicated by a hatched bar. During the first $100 \mathrm{~d}$ after surgery, the immediate deficits were assessed (period 2). In a third period, extending approximately from day 100 to 250 , parametric effects were studied. Additional data obtained at the $2^{\circ}$ spacing at the end of period 3 are shown as a third cluster of symbols (labeled 3). In a fourth period, segregation was measured after retraining. The duration of the retraining is indicated by the width of the hatched bar preceding period 4. Data obtained after retraining were labeled as period 4 , although retraining and retesting in cat 57 occurred approximately at the same time as the other animals were being tested in period 3 . 


\section{TIER I LESION \\ DOT/ANNULUS - TEXTURE}

A

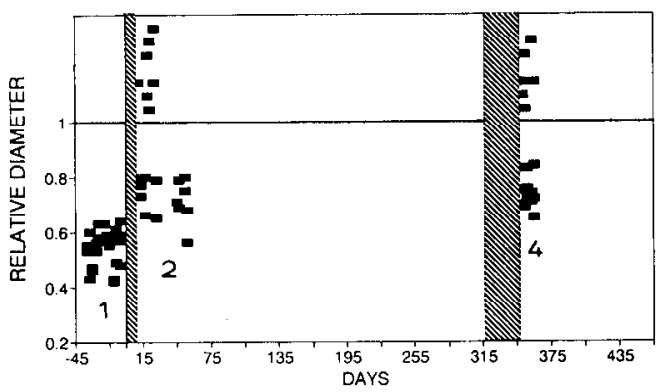

$B$
RANDOM

CAT 57

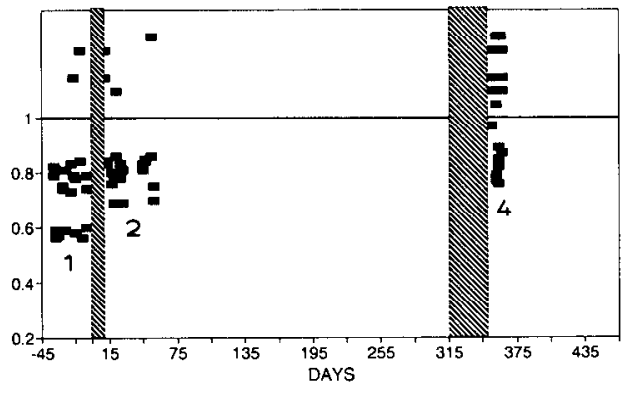

CAT 65
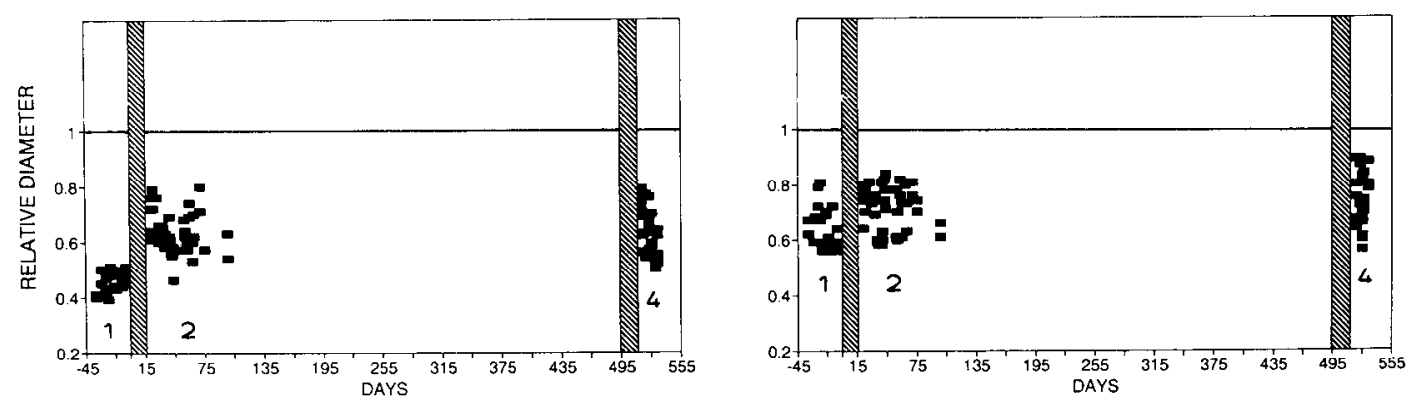

Figure 12. Segregation of dot/annulus textures after a tier I lesion. Segregation of regular $(l c f t)$ and randomized (right) dot/annulus textures before (period 1) and after (periods 2 and 4 ) the tier I lesion is shown in cats 57 and 65 . Element spacing was $1^{\circ}$. Conventions are as in Figure 11 .

ement position randomization $[F(1,24)=7.05 ; p<0.014]$. On the other hand, segregation performance after the tier II lesion (cat 64 in Fig. 16), this time measured by means of fixed stimulus measurements, was equally poor at all element spacings $[F(1$, $28)=0.92 \mathrm{~J}$. This adds to the picture that tier II lesions destroy the segregation of any texture stimulus.

\section{The effect of an added luminance cue on segregation performance}

Animals with tier II lesions were severely impaired for the two texture types used, for a wide range of stimulus parameters. The question then arose whether these animals were able to perform any detection task at all. Therefore, using the line texture, we gradually decreased the brightness of the background elements, keeping the luminance of the target elements at $24 \mathrm{~cd} / \mathrm{m}^{2}$. The resulting luminance gradient was quantified by subtracting the contrast index of the dimmer elements from the contrast index of the brighter ones. The same manipulation was carried out for two of the three cats with tier I lesions, to assess whether they would benefit similarly from the addition of the luminance gradient.

Figure 17 shows relative length thresholds expressed as a function of the strength of the luminance cue for two tier I cats and two tier II cats. Segregation performance in both lesion groups benefited from an added luminance cue, but only if relatively large cues were introduced. When no luminance cue was available (zero luminance cue), thresholds could only be measured in cat 65 using regular line textures (tier I lesion), and in cat 59 (tier II lesion). Adding the 0.1 luminance cue, which corresponds to a difference of $5 \mathrm{~cd} / \mathrm{m}^{2}$ between elements in target and background, did not cause impressive changes in segregation performance. In the two animals with tier I lesions, this luminance cue still was insufficient to reach stimulus control in the randomized condition, whereas performance in the regular condition remained unchanged in cat 65 , and only allowed limitcd segregation performance in cat 63 . The inability to exploit this considerable luminance (a $5 \mathrm{~cd} / \mathrm{m}^{2}$ difference between elements) during line texture segregation is an important finding, since it strongly suggests that the sparing of segregation between dot and annulus textures (see Fig. 12) cannot be explained by the small luminance difference between these textures (a difference between elements smaller than $0.05 \mathrm{~cd} / \mathrm{m}^{2}$ ). Similarly, the animals with tier II lesions showed no improvement (cat 64) or very limited improvement (cat 59) after addition of a $5 \mathrm{~cd} / \mathrm{m}^{2}$ luminance cue. Further increases of the luminance cue, however, showed that all animals were capable of detecting a luminancedefined target, provided this cue was strong enough. For the largest luminance cues, however, performance reached an asymptotic level that was elevated in tier II animals compared to animals with tier I ablations. Hence, those animals with intact tier II cortex benefited more from the addition of the luminance cue than those with tier $I$ areas intact.

\section{Lesion deficits after retraining: an evaluation of final deficits}

Upon finishing observation period 3 , we retrained and retested all animals during a fourth period of behavioral observations, which extended as much as 18 months after the lesion. The duration of the DEM retraining (see Materials and Methods) in 

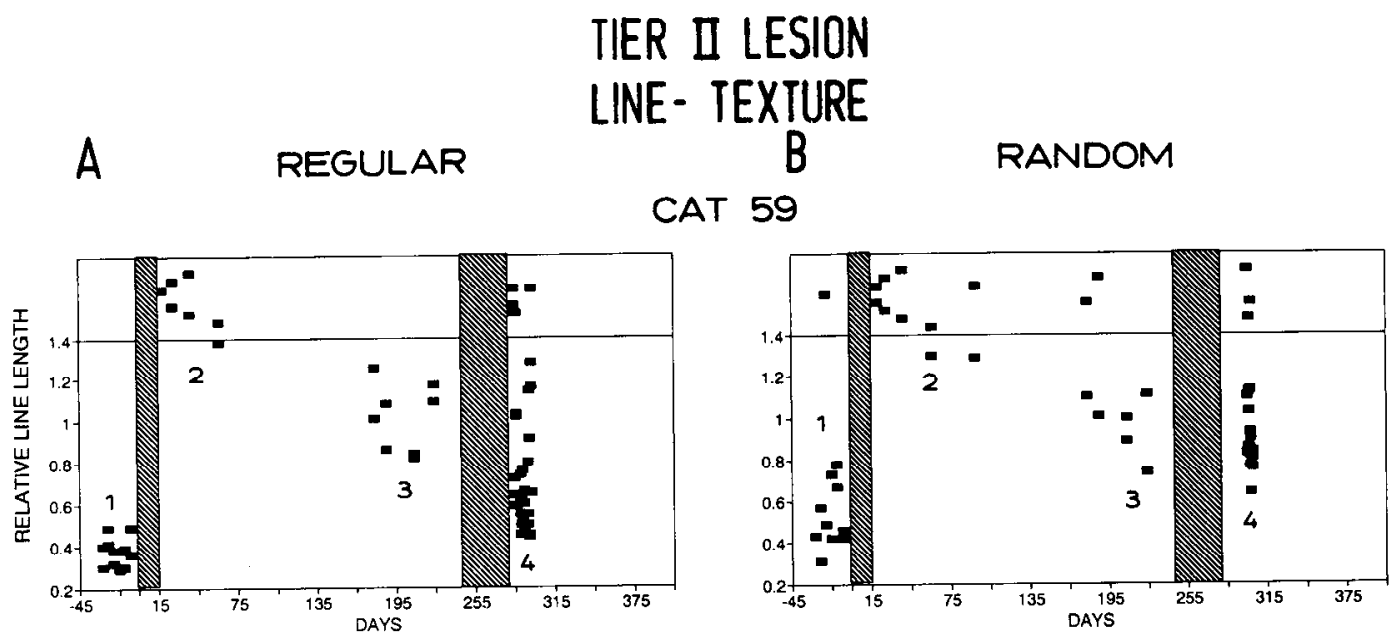

CAT 64
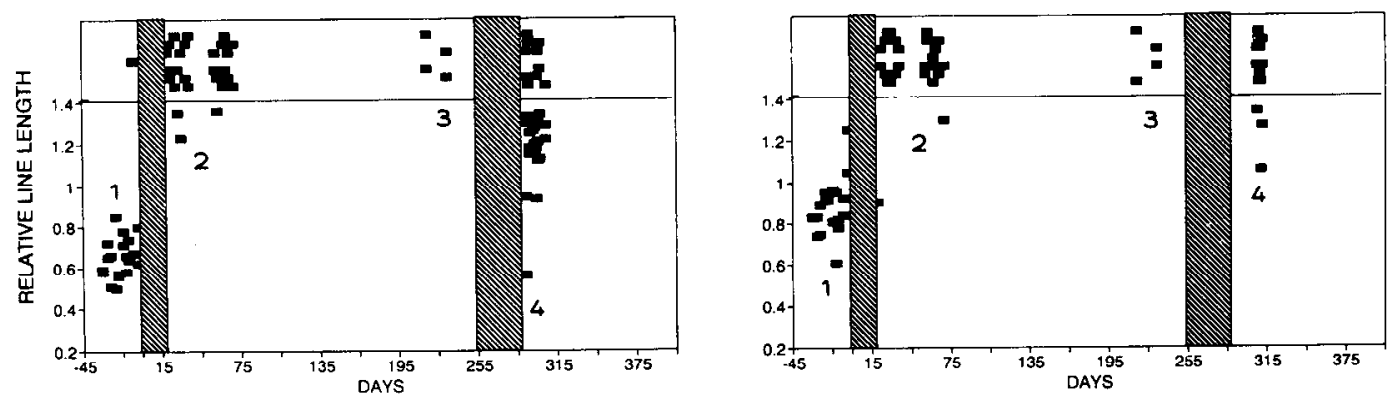

Figure 13. Line texture segregation after a tier II lesion. Segregation performance of cats 64 and 59 is plotted as a function of time before (period 1) and after (periods $2-4$ ) the tier II lesion. The stimuli used were a regular line texture (left) and a randomized line texture (right) with a $2^{\circ}$ element spacing. Conventions are as in Figure 11.

the regular condition at the standard spacing is indicated by the hatched bar following period 3 (Figs. 11-14). The retraining was ended if no significant increase in performance was observed in 10 consecutive sessions, and it lasted for a total of 22 sessions of 300 trials on average (range, 14-26). In normal animals, the same number of sessions results in clear progress, and is sometimes sufficient to bring an animal close to threshold (De Weerd et al., 1990a, 1992). In view of the limited effects of retraining

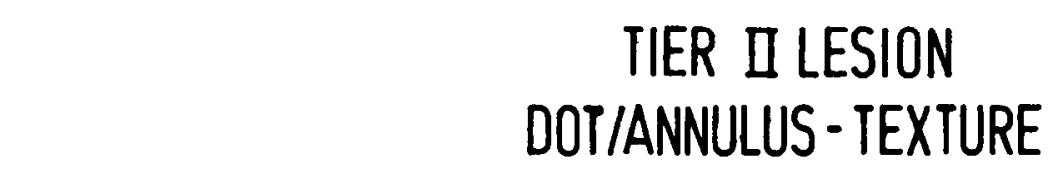

A

REGULAR

B

RANDOM

CAT 64
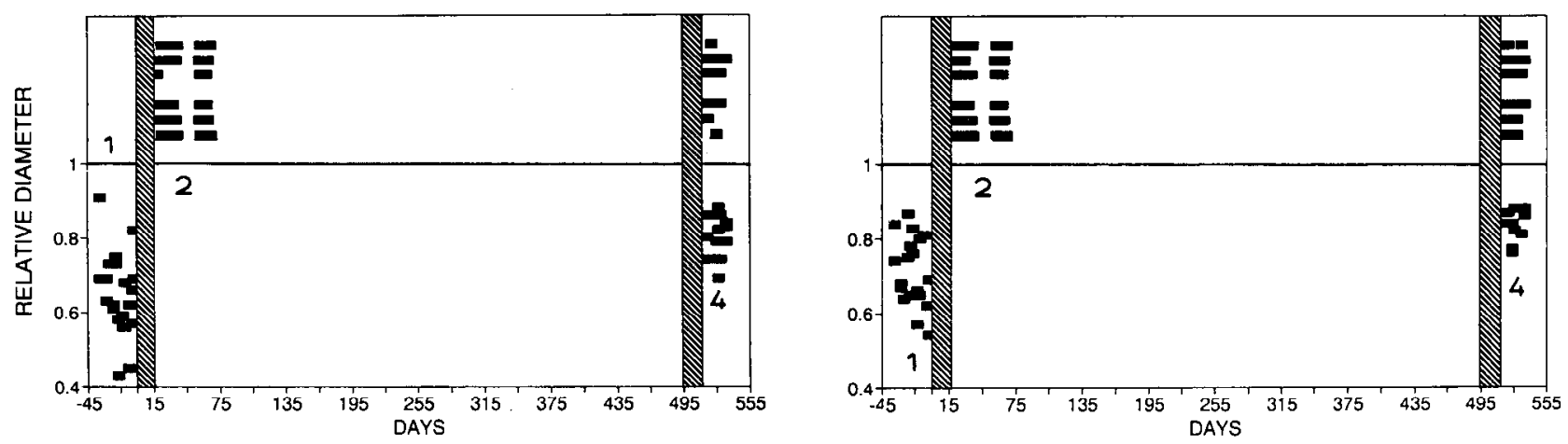

Figure 14. Dot/annulus texture segregation after a tier II lesion. Segregation of regular (top panels) and randomized dot/annulus textures is shown before (period 1) and after (periods 2 and 4) the tier II lesion in cat 64 , and was measured with the staircase method. Element spacing was $1^{\circ}$. Conventions are as in Figure 11. 

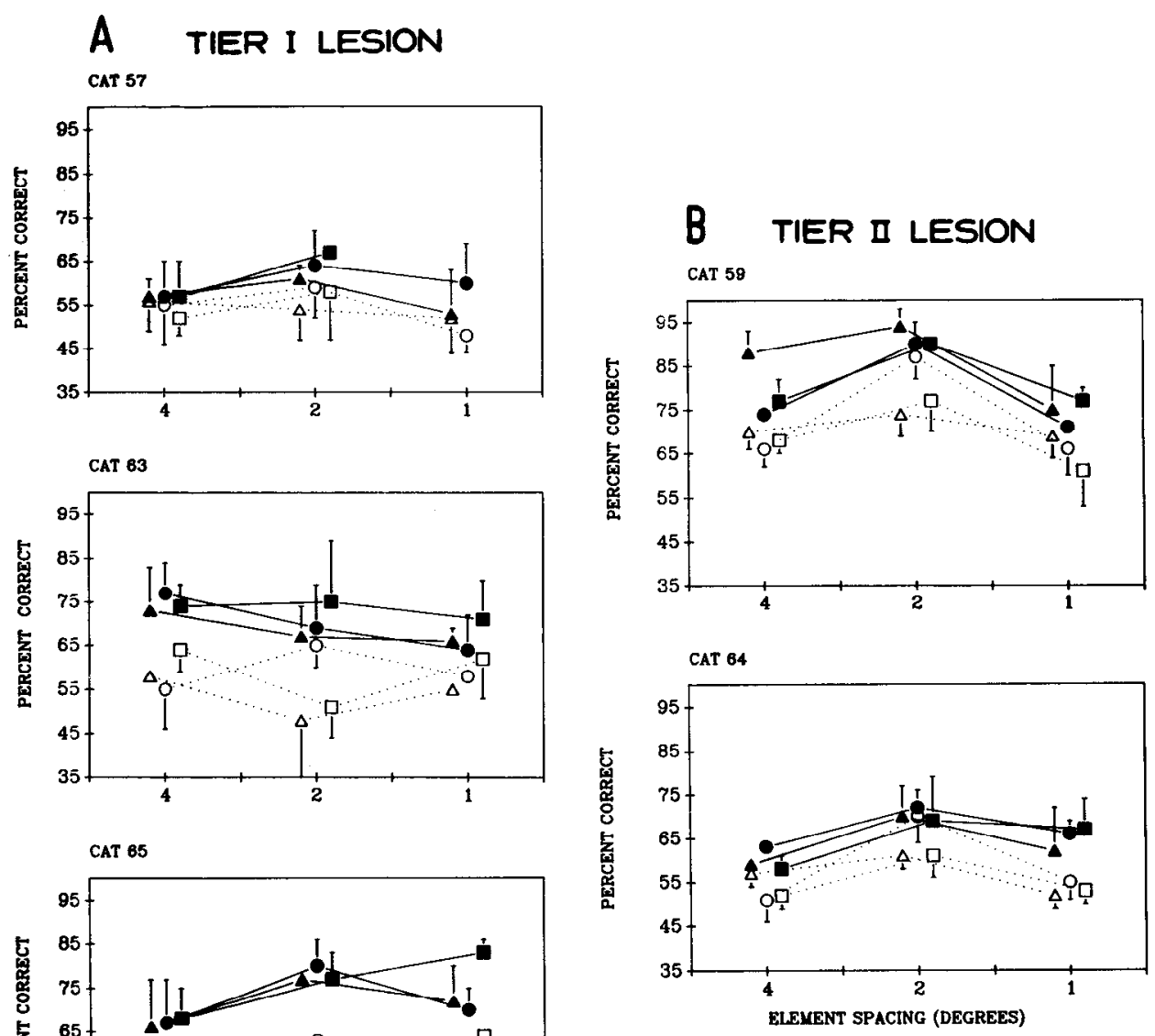

Figure 15. The effect of parametric variations on line texture segregation. Postoperative segregation measurements after tier I lesions (cats 57,63, $65)$ and tier II lesions (cats 59 and 64) are plotted as a function of element spacing, and were measured with the fixed stimulus method. Segregation is assessed using regular (solid symbols) and randomized line textures (open symbols), with line widths of 0.1 (triangles), 0.2 (circles), and $0.4^{\circ}$ (squares). Error bars represent standard deviations $(N=4)$.

in the regular condition, we decided not to retrain in the randomized condition. In addition, we assumed that if there were any training effects, performance in randomized conditions would benefit from the retraining in the regular condition, as is the case in normals (De Weerd et al., 1992). While we chose not to retrain in the randomized conditions, we did include the randomized condition in the testing after retraining. The bars following period 3 in the graphs corresponding to the randomized conditions merely indicate the absence of testing due to retraining in the regular condition. The retraining and testing at the standard spacing was followed by retraining and retesting at other spacings. The results obtained at the nonstandard spacings are not shown in Figures 10-14, but are briefly described at the end of this section.

The effect of retraining and the final lesion deficit for standard element spacings can easily be noticed by reinspecting Figure 10. Figure $10 \mathrm{~A}$ illustrates that the deficit in line texture segregation after tier I lesions remained virtually unchanged during the complete postoperative observation period, in regular as well as in randomized conditions. Only in the regular condition did we observe a slight increase in the number of staircase measurements leading to stimulus control, suggesting limited recovery in that condition only. In particular, the number of measurements with stimulus control in the regular condition in observation period 4 was significantly larger compared to period $2\left(\chi^{2}=16.38 ; \mathrm{df}=1 ; p<0.001\right)$. This increase seems to reflect spontaneous recovery that already had occurred before retraining (period 3), rather than the effect of retraining itsclf. Thresholds after retraining in both conditions of element position randomization typically remained larger than 1.20.

Despite the limited effect of the tier I ablation on the segregation of dot/annulus textures, which might suggest retraining to be more effective, there was also in this case no sign of relearning in either the regular or the randomized conditions (Fig. 10B). Thus, although the lesion deficit in segregating the dot/annulus stimulus was small, it was as stable as the large deficit seen for line textures.

On the other hand, a comparison of line texture segregation in periods 2 and 4 after tier II ablations revealed a definite recovery (Fig. 10C), which expressed itself both in an increase in the number of measurements with stimulus control $\left(\chi^{2}=\right.$ 43.66, $\mathrm{df}=1, p<0.001$ in the regular condition; $\chi^{2}=14.26$, $\mathrm{df}=1, p<0.001$ in the randomized condition), and in a decrease of relative length thresholds. The improved segregation performance of cat 59 in period 4 reflects the effects of both spontaneous recovery and retraining, whereas in cat 64 it only reflects the effect of retraining (Fig. 13). Thresholds obtained from staircase measurements with stimulus control remained significantly elevated compared to preoperative performance in regular $(t=$ $-5.47, \mathrm{df}=89, p<0.001)$ and randomized conditions $(t=$ 3.04, $\mathrm{df}=42, p<0.004)$.

Also, the segregation of dot/annulus textures showed recovery 


\section{TIER I LESION}

\section{CAT 65}

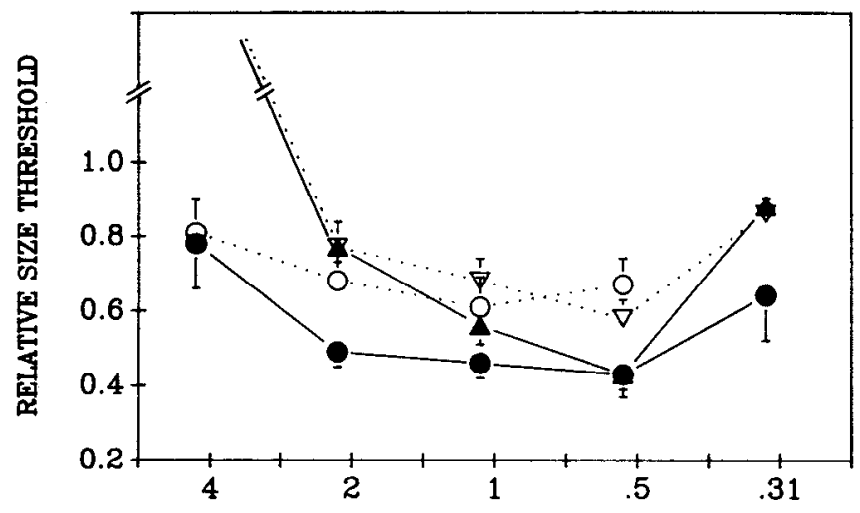

TIER I LESION

\section{CAT 64}

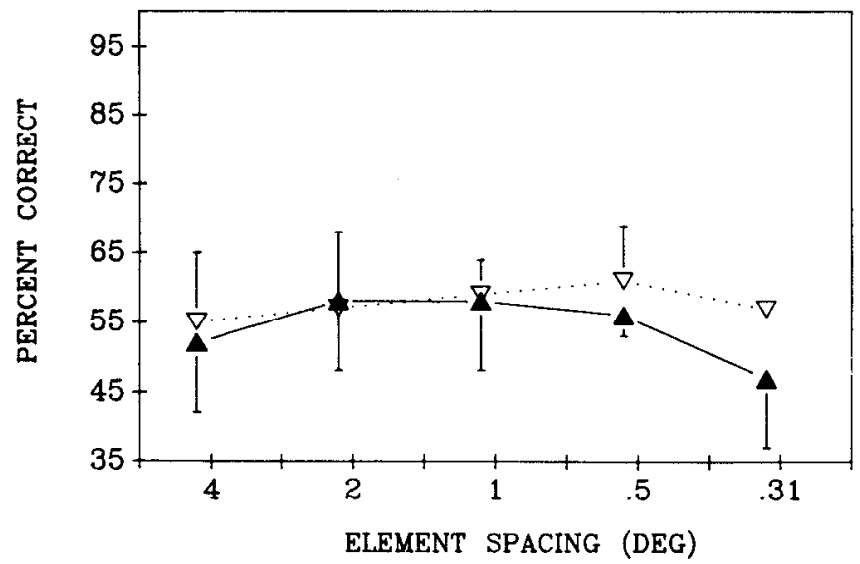

Figure 16. The effect of parametric variations on dot/annulus texture segregation. Segregation of dot/annulus texture stimuli plotted as a function of spacing in cat 65 (tier I lesion) and cat 64 (tier II lesion). Performance was measured with the staircase procedure in cat 65 , and with the fixed stimulus procedure (relative annulus diameter of 0.95 ) in cat 64. Open symbols represent values obtained in the randomized condition, and solid symbols refer to the regular condition. Circles in the upper graph represent preoperative performance; triangles indicate postoperative measurements. In cat 65 , no postoperative thresholds could be determined at the $4^{\circ}$ element spacing. Error bars represent standard deviations $(N=6)$.

after tier II lesions. The number of thresholds with stimulus control increased significantly after retraining $\left(\chi^{2}=14.57, \mathrm{df}=\right.$ $1, p<0.001$ in regular condition; $\chi^{2}=9.87, \mathrm{df}=1, p<0.002$ in randomized condition). In addition, the staircases obtained after retraining in the randomized condition were not significantly different from preoperative ones $(M=37 ; p<0.068)$. The results obtained at the standard spacings taken together show that there was significant recovery after the tier II ablations, in contrast to the virtually stable performance after tier I lesions.

All cats were also retrained in the regular condition at one or more spacings different from the standard spacings, for an average of 20 sessions per element spacing (range, 16-23 sessions). Subsequent testing results were in agreement with our findings at standard spacings. Cats with tier I lesions (57, 63, and 65) reached stimulus control in $40 \%$ of the measurements at spac- ings of $1^{\circ}$ and $4^{\circ}$, as opposed to $3 \%$ in the randomized condition (pooled over cats; compare with Fig. 15). Using dot/annulus textures of a $2^{\circ}$ element spacing resulted in a 0.75 relative diameter threshold, averaged over 13 staircases with stimulus control obtained from 20 measurements. These results indicate a strong deficit in line texture segregation after tier I lesions, with considerable sparing, however, of dot/annulus texture segregation. On the other hand, the strong recovery in the segregation of regular line textures at the standard spacing in cat 59 (tier II lesion) was also reflected in its performance at $4^{\circ}$ and $1^{\circ}$ spacings, with thresholds of 1.03 and 1.20 , respectively, and percentages of stimulus control exceeding $85 \%$. Performance with randomized textures, however, was less efficient, especially for the $1^{\circ}$ spacing in which stimulus control was never reached. The limited recovery at standard spacings in cat 64 was not reflected in good residual performance at other spacings, for either of the two texture types used (compare with Figs. 15, 16).

\section{Discussion}

Our results show that texture segregation relies on tier I as well as on tier II cortical areas. The effects of the two lesions, however, differed in two major respects. First, the deficit caused by tier I lesions was much less pronounced for dot/annulus textures than for line textures, whereas tier II ablations resulted in deficits equally strong for both texture stimuli. Second, we observed more recovery after tier II lesions than after tier I lesions. Using line textures, we also observed some remarkable similarities after lesions of tier I and tier II areas. First, residual segregation after either ablation occurred only at increased relative lengths, at which alternative cues might supplement the texturc difference. Second, the lesion deficits were large for any combination of stimulus settings. Third, postoperative segregation performance could be improved by superimposing a large luminance cue upon the texture difference.

The behavioral deficits varied little among the three cats with tier I lesions (i.e., ablations of areas 17 and 18), which fits with the comparable extent of the lesions in these animals. The somewhat larger deficit in cat 63 may be explained by the significant bilateral involvement of area 19 in that animal's lesion. There was more variation in the extent of tier II lesions, which targeted areas 7, 19, 20a, 21a, 21 b, AMLS, and PMLS. More of 19 was spared in cat 59 than in cat 64 , whereas some sparing of $21 \mathrm{~b}$ was found in the right hemisphere of cat 64 . Importantly, any similarity in the effects of tier I and tier II lesions cannot be due to indirect dysfunctioning of areas 17 and 18 after tier II lesions. LGN laminae $A$ and $A 1$ were anatomically intact after the ticr II lesions, which shows that the major afferents to areas 17 and 18 were spared, a conclusion supported by physiological recordings demonstrating that neurons in areas 17 and 18 were functioning normally after tier II lesions. In this respect, it is also worth mentioning that bar orientation discrimination after tier II ablations was unaffected even at short lengths and low contrasts, suggesting that acuity and contrast sensitivity are unimpaired after this lesion (Vandenbussche et al., 1989; Sprague et al., 1993).

Few studies have been devoted to the assessment of texture segregation after lesions in visual cortical areas. In one such study, reported in abstract form, Aglioti et al. (1988) claimed that lesions of areas 17 and 18 have little effect on texture segregation in the cat. Our results show that the effect of tier I lesions depends on the texture stimulus used, and therefore, it 

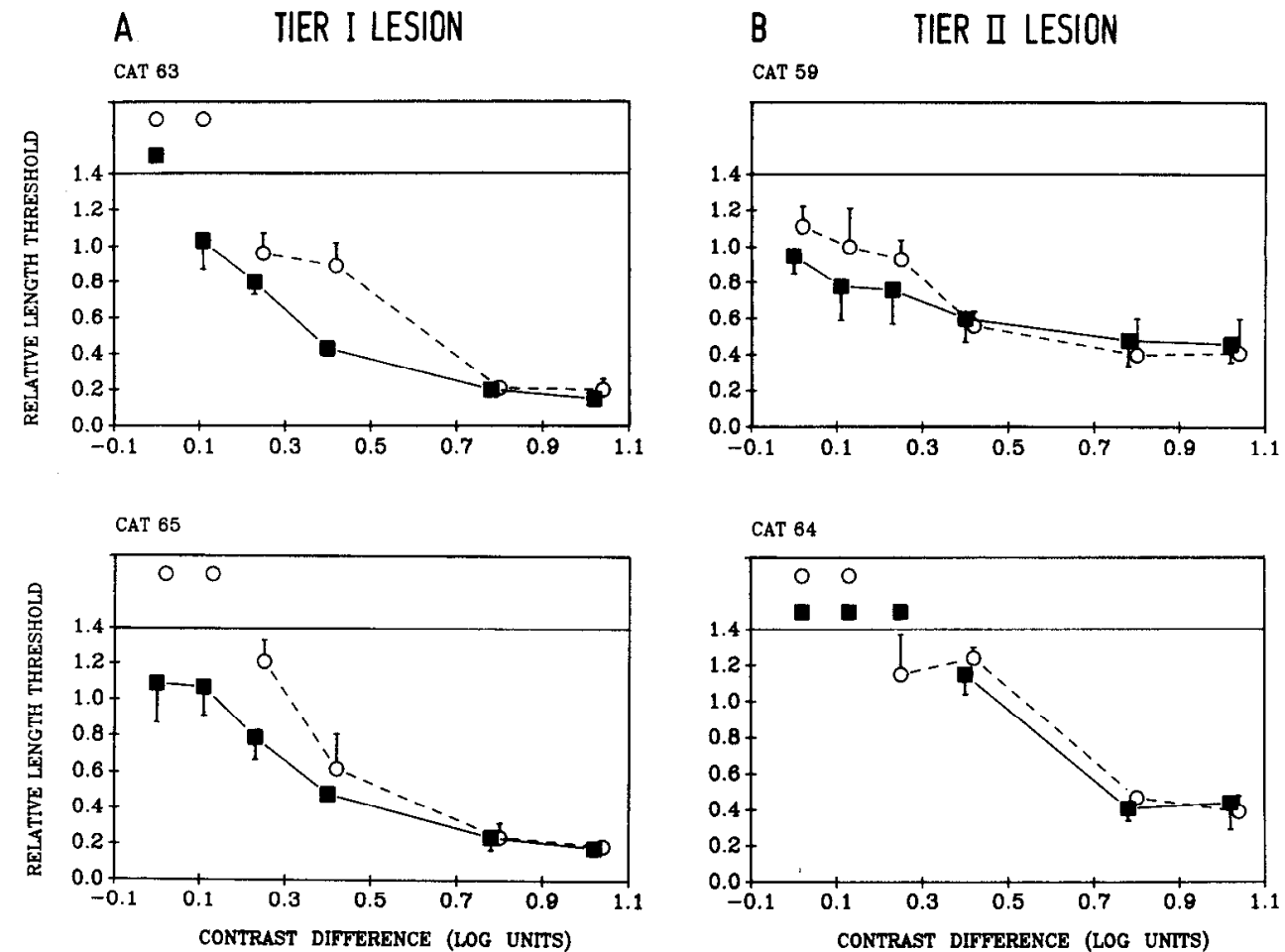

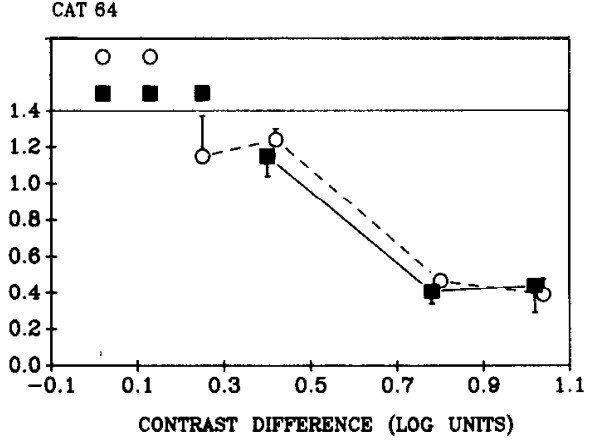

Figure 17. The effect of superimposing a luminance cue on the orientation difference in line textures. Relative length thresholds obtained with line textures are plotted as a function of local contrast difference between target and background elements. Thresholds were measured in two animals with tier I ablations and in two animals with tier II ablations. Solid symbols show thresholds obtained in the regular condition; open symbols show thresholds obtained in the randomized condition. Each symbol represents four measurements of 75 trials. Symbols were placed above the 1.4 ceiling to indicate instances in which the animals did not reach stimulus control in a total of 300 trials from four measurements. Symbols below the ceiling indicate the average of four staircase measurements with stimulus control. Error bars represent standard deviations $(N=4)$. is likely that Aglioti et al.'s (1988) result was determined by particular characteristics of their stimulus. In another study, Wilkinson (1990) removed areas 17, 18, and 19 in kittens shortly after birth, and started testing them at $30 \mathrm{~d}$ of age. Severe deficits in texture segregation appeared, whereas pattern and brightness discrimination and acuity were comparable to unoperated litter mates. However, Wilkinson's (1990) findings cannot easily be compared with those of Aglioti et al. (1988) or ours, since there was significant damage in lateral suprasylvian areas in addition to the lesion in areas 17,18 , and 19.

The ablation of tier $I$ areas severely impaired the segregation of line textures, but largely spared the segregation of dot and annulus textures. In contrast to the line texture stimuli, there was a small luminance cue available in the dot/annulus textures, and therefore one could argue that the above differential result was due to the efficient use of the small luminance cue in the dot/annulus textures. However, we have shown (Fig. 17) that the magnitude of a luminance cue that could explain the considerable sparing of segregation observed with dot/annulus texlures has to be much larger than the small luminance cue available in these textures. Therefore, the above-mentioned differential result cannot be attributed to luminance cues in dot/ annulus textures. Interestingly, normal animals are also quite inefficient in using small luminance differences as an additional cue during texture segregation (Vandenbussche et al., 1993). Finally, the losses in contrast sensitivity and acuity reported after tier I ablations (Berkley and Sprague, 1979; Pasternak et al., 1990) argue against the possibility that a small luminance cue would significantly improve any type of texture segregation. Rather, the sparing of dot/annulus texture segregation may be due to the fact that dot and annulus textures still match some filtering capacities of the visual system remaining after a tier I ablation. The segregation of dot and annulus textures probably relies on differences in spatial frequency, which could be detected by the dLGN, and does not require orientation aniso- tropic filtering by units in tier I areas. Nothdurft (1990b) has in fact reported that cat LGN neurons respond to borders between fine and coarse textures of equal mean luminance. These border responses were maximal for texture element spacings near the resolution limit of the cell. In this respect, it is worth noting that the reduction in acuity after tier $I$ lesion (Berkley and Sprague, 1979; Pasternak et al., 1990) and the sparing of the dot/ annulus segregation after the same lesion, are not in contradiction with the use of spatial frequency in the dot/annulus segregation. Indeed, the annulus texture, which contains the higher spatial frequency, does not need to be resolved in order to achieve segregation from the dot texture characterized by a lower spatial frequency content (De Weerd et al., 1992). Note that the restoration of the segregation between line textures after making target elements considerably brighter than background elements could reflect the fact that under these conditions the filtering by the dLGN is sufficient for the subsequent comparison process, much in the same way as filtering by the LGN might be sufficient when the relevant cue is a difference in spatial frequency. In particular, the local differences in neural activity in the LGN due to luminance differences in the textures (Nothdurft, 1990b) could be used directly by the comparison stage to achieve segregation between different regions of the image.

Our finding that some textures can be segregated after tier I ablations implies that the comparison stage, which we assume to be common to all sorts of texture, is located outside tier I cortex. Other results, discussed below, strongly suggest that one or more areas belonging to tier II cortex are involved in the comparison stagc. Apart from strong inputs from areas 17 and/ or 18 , all tier II areas receive direct projections from at least one subdivision of the dLGN other than the $A$ and $A 1$ layers (i.e., C, Cl-C3, MIN, or GW) (Rosenquist, 1985). It is these projections that could sustain the segregation between dot and annulus textures and between texture regions made of bright versus darker elements after removal of tier I cortex. Third, the 
dependency of line texture segregation on tier I areas and the finding that areas 17 and 18 do not intervene in the comparison stage are in agreement with the predominant role of tier I cortex in the filtering of the retinal image, a conclusion we had derived earlier from the effects of tier I ablations on bar orientation discrimination (Orban et al., 1990; Vandenbussche et al., 1991).

On the other hand, the effects of tier II ablations reported in the present study contrast with those we obtained using a bar orientation discrimination task. Tier II lesions dramatically impaired line texture segregation, whereas bar orientation discrimination remained intact after similar lesions (Vandenbussche et al., 1989). This is particularly interesting since the elementary unit of the line texture stimulus is the same as in the discrimination task (a bar). Thus, it appears that tier II areas provide the extra computations required to use differences in line orientation in a segregation task, computations that are not necessary to judge the orientation of the same line when shown in isolation. Interestingly, tier II lesions completely wiped out the segregation of both regular and randomized textures, whereas tier I lesions allowed some residual performance in regular conditions. Lesions in tier II cortex also severely impaired the segregation of dot and annulus textures as opposed to the sparing after tier I lesions. Furthermore, addition of a luminance gradient to the texture difference proved to be less beneficial for animals with tier II ablations than for animals with tier I ablations. The global scope of the deficits caused by tier II lesions is in agreement with the idea that tier II areas house general comparison processes underlying the segregation of a variety of textures. Our lesion results do not indicate what these processes are, but they may involve both the grouping of similar elements and the distinction of dissimilar elements. Hughes and Sprague (1986) provided evidence for a role of tier II areas in the grouping of similar orientations, and some proposals for the underlying processes have been put forward (e.g., Rubenstein and Sagi, 1990). However, in view of the crucial importance in texture segregation of elements near the border (Nothdurft, 1985; Landy and Bergen, 1991; De Weerd et al., 1992), an important process taking place in tier II areas might be similar to the one proposed by Orban and Gulyás (1988) for kinetic boundaries. According to their scheme, local differences are detected by antagonistic surround units after which these differences are spatially integrated by units representing the kinetic or texture boundary.

The above scheme is attractive, but we must also consider an alternative explanation, related to the involvement of area 7 in the tier II lesions. Olson (1991) showed that the strength of neuronal responses in area 7 to a particular stimulus predicts whether that stimulus will capture attention. Spontaneous eye movements towards the stimulus were used as an indicator of attention. The deficit seen in our study after tier II ablations, thus, could be due to an inability to use the output of the comparison stage in directing attention towards the relevant stimulus (the change in texture). If this were the case, there is no need to place the comparison stage in tier II areas; both stages of texture segregation might be implemented in tier I areas. However, two points argue against this explanation. First, if a general comparison stage were located in tier I areas, one would expect segregation of different types of textures to be equally affected after tier I lesions. Instead, texture segregation seems to depend on whether or not the texture stimulus fits the filtering characteristics of the residual visual system spared by the lesion. Second, the idea that cats are unable to direct attention towards a salient stimulus after removal of area 7 is weakened by the observation that line textures could be segregated if their elements were of a different brightness.

From the previous reasoning, we conclude that the two serial stages put forward in computational models of texture segregation (see introductory remarks) are implemented in different groups of visual cortical areas. In particular, our data support the notion that, in normal animals, tier II lesions carry out a comparison step on the basis of an image filtered by tier I areas. This model has two important implications. First, it argues against a significant contribution of direct thalamic input to tier II areas in normal segregation performance. The retinal input reaching tier II areas via the superior colliculus and the lateral posterior pulvinar nuclei has been proposed to explain nearnormal discrimination of large patterns and shapes presented on a homogeneous background after removal of areas 17 and 18 (Wood et al., 1974; Baumann and Spear, 1977; Sprague et al., 1977, 1985; Berkley and Sprague, 1979; Berlucchi et al., 1979). However, the devastating and enduring effect of tier I lesions on line texture segregation shows the irrelevance of this pathway for the line texture stimulus. The small loss of segregation of dot/annulus textures after tier I lesions also shows for this stimulus that direct thalamic input to tier II areas, while important, is insufficient to maintain optimal performance. Second, the involvement of tier II areas in texture segregation raises reservations against the notion that texture segregation is wholly an "early" process in vision, since all cortical areas included in tier II belong to a second level of the cortical hierarchy (Rosenquist, 1985).

In spite of differences in the layout of the visual systems of cats and primates (Orban, 1984; Rosenquist, 1985; Desimone and Ungerleider, 1989), we suggest that some higher-order areas in the primates, possibly belonging to the ventral pathway (Ungerleider and Mishkin, 1982; Desimone and Ungerleider, 1989), might play a role in texture segregation similar to the one of tier II cortex in the cat. The above-suggested similarity of neural mechanisms for texture segregation in primates and cats remains to be demonstrated. However, the qualitative resemblances of performance of cats and humans in basic visual acuity tasks have led scveral authors to arguc convincingly that the neural mechanisms intervening in such tasks are similar in both species (Uhlrich et al., 1981; Petry et al., 1984; Berkley, 1989; see also: De Weerd et al., 1990b; Pasternak and Horn, 1991). The close parallels in texture segregation in humans (Nothdurft, 1985, 1990a; Landy and Bergen, 1991) and cats (De Weerd et al., 1992) suggests to us that their neural circuitries underlying texture segregation might also show significant similarities. Note that the recent discovery of cells sensitive to orientation contrast in area V1 in the monkey (Knierim and Van Essen, 1992) does not exclude the possibility that also in this species higher-order areas intervene in texture segregation. A number of authors, including Knierim and Van Essen (1992), have considered the possibility that complex properties in lower-order areas depend on feedback from higher-order areas (e.g., Finkel and Edelman, 1989; Nakayama et al., 1990; Okajima, 1991).

The term "tier II" refers to a large number of cortical areas. Probably, these areas do not intervene equally in texture segregation. The tier II cortex included areas AMLS and PMLS. Visual areas in LS have been implicated in different sorts of motion processing and optic flow analysis (Spear and Baumann, 1975; Rauschecker et al., 1987; Toyama et al., 1990; Rudolph and Pasternak, 1992; Yin and Greenwood, 1992a,b). It is pos- 
sible then that the critical areas intervening in the segregation of a stationary stimulus lie outside LS altogether. Our data provide direct evidence against the involvement of AMLS in texture segregation since it was spared in both cats 59 and 64 . Similarly, area $21 \mathrm{~b}$ is unlikely to play a significant role, in view of the large unilateral sparing of that area in cat 64 . This leaves areas $19,20 \mathrm{a}$, and $21 \mathrm{a}$ as possible candidates. On the basis of the anatomical connections of these three areas, we propose that they may act as a unit in texture segregation. All three areas receive major projections from areas 17 and 18 . In addition, all three also receive from layers $\mathrm{C} 1-\mathrm{C} 3$ of the dLGN, and from all three subdivisions of the posterior pulvinar complex (Rosenquist, 1985). Finally, areas $20 \mathrm{a}$ and 21 a send major reciprocal projections to area 19. Thus, in normal animals, areas 19, 20a, and $21 \mathrm{a}$ might closely collaborate during the comparison of textures on the basis of filtered input from areas 17 and 18 .

Two comments must be made, however, with respect to the function of areas 19 and $21 \mathrm{a}$. The large number of end-stopped units in area 19 (Hubel and Wiesel, 1965; Duysens et al., 1982; Tanaka et al., 1987; Saito et al., 1988), which keep their modest orientation selectivity after removal of tier I areas (Lepore et al., 1991), may also suggest a role of this area in supplementing areas 17 and 18 in the filtering of especially the line textures. On the other hand, tier I ablations leaving arca 19 intact caused profound deficits in both line texture segregation (cats 57 and 65 ) and bar orientation discrimination (Vandenbussche et al., 1991), which shows that this area contributes little to orientation filtering. Rather, results of Hubel and Wiesel (1965) and Saito et al. (1988) suggest that area 19 intervenes in the detection of corners and end points, and our behavioral results support this possibility. Indeed, the two animals of this study with limited involvement of area 19 (cats 57 and 65) were the only ones to show some residual performance immediately after tier I ablation (Fig. 11), possibly because they could use the alternative cues available at large relative line lengths (crosses and corners) as a cue in the detection task. Hence, area 19 may perform a specialized type of filtering of the retinal image, separate from orientation filtering, and also separate from a possible role in the comparison stage. Second, recent evidence that neurons in area 21 a prefer long bars as a stimulus (Mizobe et al., 1988; Henry et al., 1991) may seem at odds with a role in the segregation of textures made of short elements. However, it is not necessary for the units that compare a local parameter over space to be sensitive for each individual element (Marcar et al., 1992). The preference in area $21 \mathrm{a}$ for long bars may represent the constraint that only differences in a local parameter extending over a sufficient distance are signalled as a relevant discontinuity, which is in line with a possible role of area $21 \mathrm{a}$ in the comparison stage.

A remarkable aspect of residual performance was the virtual absence of recovery after tier I lesions compared to a definite, though partial, recovery after tier II lesions. The absence of recovery after tier I lesions fits the interpretation of this lesion's effect as a hardware deficit (a loss of a number of filters). The recovery after tier II lesions, on the other hand, suggests that visual areas outside tier II areas, or remaining parts of tier II cortex, can reorganize to substitute for the lost comparison stage. Residual performance initially was possible only for element sizes close to the element spacing, and therefore was likely to be based on alternative cues at the border between textures. However, the significant recovery in cat 59 , in which small element sizes could be used, suggests that the areas remaining after a tier II ablation can reorganize to implement a nearnormal comparison stage.

The combination of the present study with our previous study on bar orientation discrimination (Vandenbussche et al., 1991) is one more demonstration of the necessity to manipulate the complexity of the stimulus in order to unravel the functions of higher-order visual areas. We suggest that one of the functions of tier II areas consists of the comparison between textures based on filtered input provided by tier I areas.

\section{References}

Aglioti S, Berlucchi G, Corbetta M, Antonini A (1989) Texture discrimination and perception of cognitive contours in cats before and after cortical ablations. Soc Neurosci Abstr 14:1252.

Baumann TP, Spear PD (1977) Role of the lateral suprasylvian visual area in behavioral recovery from effects of visual cortex damage in cats. Brain Res 138:445-468.

Beck J (1982) Textural segmentation. In: Organisation and representation in perception (Beck J, ed), pp 285-317. Hillsdale, NY: Erlbaum.

Bergen JR, Adelson EH (1988) Early vision and texture perception. Nature 333:363-364.

Bergen JR, Julesz B (1981) Discrimination with brief inspection times. J Opt Soc Am 71:1570.

Berkley MA (1970) Visual discriminations in the cat. In: Animal psychophysics: the design and conduct of sensory experiments (Stebbins W, ed), pp 231-247. New York: Appleton Century-Crofts.

Berkley MA (1989) Integration for vernier acuity in the cat: comparative aspects of cortical scaling. Vis Neurosci $2: 473-482$.

Berkley MA, Sprague JM (1979) Striate cortex and visual acuity functions in the cat. J Comp Neurol 187:697-702.

Berlucchi G, Sprague JM, Antonini A, Simoni A (1979) Learning and interhemispheric transfer of visual pattern discriminations following unilateral suprasylvian lesions in split-chiasm cats. Exp Brain Res 34: 551-574.

Brady M, Grimson WEI (1981) The perception of subjective surfaces. MIT A.I. Memo 666.

Desimone R, Ungerleider LG (1989) Neural mechanisms of visual processing in monkeys. In: Handbook of neuropsychology, Vol 2 (Boller F, Grafman J, eds), pp 267-299. New York: Elsevier.

Devos M, Orban GA (1990) Modeling orientation discrimination at multiple reference orientations with a neural network. Neural Comput 2:152-161.

De Weerd P, Vandenbussche E, Orban GA (1990a) Speeding up visual discrimination learning in cats by differential exposure of positive and negative stimuli. Behav Brain Res 36:1-12.

De Weerd P, Vandenbussche E, Orban GA (1990b) Bar orientation discrimination in the cat. Vis Neurosci 4:257-268.

De Weerd P, Vandenbussche E, Orban GA (1992) Texture segregation in the cat: a parametric study. Vision Res 32:305-322.

Duysens J, Orban GA, van der Glas HW, de Zegher FE (1982) Functional properties of area 19 as compared to area 17 of the cat. Brain Res 231:279-291.

Finkel LH, Edelman GM (1989) Integration of distributed cortical systems by reentry: a computer simulation of interactive functionally segregated visual areas. J Neurosci 9:3188-3208.

Flandrin JM, Courjon JH, Orban GA, Sprague JM (1992) Longterm impairment of cat optokinetic nystagmus following visual cortical lesions. Exp Brain Res 88:594-608.

Fogel I, Sagi D (1989) Gabor filters as texture discriminator. Biol Cybern 61:103-113.

Henry GH, Wieniawa-Markiewicz E, Wimborne B, Levick WR (1991) Area $21 \mathrm{a}$ in the cat and the detection of binocular orientation disparity. Soc Neurosci Abstr 17:442.

Hubel DH, Wiesel TN (1965) Receptive fields and functional architecture in two nonstriate visual areas (18 and 19) of the cat. J Neurophysiol 28:229-289.

Hughes HC, Sprague JM (1986) Cortical mechanisms for local and global analysis of visual space in the cat. Exp Brain Res 61:332-345.

Julesz B (1981) A theory of preattentive texture discrimination based on first-order statistics of textons. Biol Cybern 41:131-138.

Julesz B (1986) Texton gradients: the texton theory revisited. Biol Cybern 54:245-251.

Julesz B, Bergen JR (1983) Textons, the fundamental elements in 
preattentive vision and the perception of textures. Bell Syst Tech $\mathrm{J}$ 62:1619-1645.

Kirk R (1968) Experimental design procedures for the behavioral sciences. Belmont: Brooks Cole

Knierim JJ, Van Essen DC (1992) Neuronal responses to static texture patterns in area V1 of the alert macaque monkey. J Neurophysiol 67: 961-980.

Landy MS, Bergen JR (1991) Texture segregation and orientation gradient. Vision Res 31:679-691.

Lepore F, Roux L, Richer L, Ptito M, Guillemot JP (1991) Orientation tuning and directional selectivity of cells in area 19 after lesion of areas 17 and 18 of the cat. Invest Ophthalmol Vis Sci 32:908.

Marcar VL, Raiguel SE, Xiao D, Maes H, Orban GA (1992) Do cells in area $\mathrm{V} 2$ respond to the orientation of kinetic boundaries? Soc Neurosci Abstr 18:1275.

Marr D (1982) Vision. A computational investigation into the human representation and processing of visual information. San Francisco: Freeman

Mizobe K, Itoi M, Kaihara T, Toyama K (1988) Neuronal responsiveness in area 21a of the cat. Brain Res 438:307-310.

Nakayama K, Shimojo S, Ramachandran VS (1990) Transparcncy: relation to depth, subjective contours, luminance, and neon color spreading. Perception 19:497-513.

Nothdurft HC (1985) Sensitivity for structure gradient in texture discrimination tasks. Vision Res 25:1957-1968.

Nothdurft HC (1990a) Texture segmentation is based on local dissimilarities. Invest Ophthalmol Vis Sci 31:561.

Nothdurft HC (1990b) Texture discrimination by cells in the cat lateral geniculate nucleus. Exp Brain Res 82:48-66.

Okajima K (1991) A recurrent system incorporating characteristics of the visual system: a model for the function of backward neural connections in the visual system. Biol Cybern 65:235-241.

Olson CR (1991) Area 7 and the spontaneous capture of attention by unexpected stimuli. Soc Neurosci Abstr 17:1282.

Orban GA (1984) Neuronal operations in the visual cortex. Berlin: Springer.

Orban GA, Gulyás B (1988) Image segregation by motion: cortical mechanisms and implementation in neural networks. In: Neural computers (NATO ASI series F) (Eckmiller R, von der Malsburg C, eds), pp 149-158. Berlin: Springer.

Orban GA, Kennedy H, Maes H (1981a) Response to movement of neurons in areas 17 and 18 of the cat: velocity sensitivity. J Neurophysiol 45:1043-1058.

Orban GA, Kennedy H, Maes H (1981b) Response to movement of neurons in areas 17 and 18 of the cat: direction sensitivity. J Neurophysiol 45:1059-1073

Orban GA, Vandenbussche E, Sprague JM, De Weerd P (1990) Orientation discrimination in the cat: a distributed function. Proc Nat Acad Sci USA 87:1134-1138.

Otsuka R, Hassler R (1962) Uber Aufbau und Gliederung der corticalen Sehsphäre bei der Katze. Arch Psychiatr Zeitschr Neurol 203: 212-234.

Palmer LA, Rosenquist AC, Tusa RJ (1978) The retinotopic organization of lateral suprasylvian visual areas in the cat. J Comp Neurol 177:237-256.

Pasternak T, Horn K (1991) Spatial vision of the cat: variation with eccentricity. Vis Neurosci 6:151-158.

Pasternak T, Zurawska I, Maunsell JHR (1990) Lesions of area 17 in the cat reduce sensitivity to high but not low spatial frequency targets. Soc Neurosci Abstr 16:962.

Petry HM, Fox R, Casagrande VA (1984) Spatial contrast sensitivity of the tree shrew. Vision Res 24:1037-1042.

Rauschecker JP, von Grünau MW, Poulin C (1987) Centrifugal organization of direction preferences in the cat's lateral suprasylvian visual cortex and its relation to flow field processing. $J$ Neurosci 7:943-958.

Rosenquist AC (1985) Connections of visual cortical areas in the cat. In: Cerebral cortex, Vol III (Peters A, Jones EG, eds), pp 81-117. New York: Plenum.

Rubenstein BS, Sagi D (1990) Spatial variability as a limiting factor in texture-discrimination tasks: implications for performance asymmetries. J Opt Soc Am A 7:1632-1643.

Rudolph KK, Pasternak T (1992) Lesions of lateral suprasylvian cortex in the cat reveal deficits in the perception of global motion. Soc Neurosci Abstr 18:1034.

Sagi D (1990) Detection of an orientation singularity in Gabor textures: effect of signal density and spatial-frequency. Vision Res 30:13771388.

Saito H, Tanaka K, Fukada Y, Oyamada H (1988) Analysis of discontinuity in visual contours in area 19 of the cat. J Neurosci 8:11311143.

Sanderson KJ (1971) The projection of the visual field to the lateral gcniculatc and medial interlaminar nuclei in the cat. $J$ Comp Neurol 143:101-118.

Sanides F, Hoffmann J (1969) Cyto- and myeloarchitecture of the visual cortex of the cat and of the surrounding integration cortices. $J$ Hirnforsch 11:79-104.

Schiller P, Logothetis NK, Charles ER (1990) Role of the color-opponent and broad-band channels in vision. Vis Neurosci 5:321-346.

Spear PD, Baumann TP (1975) Receptive field characteristics of single neurons in lateral suprasylvian visual area of the cat. J Neurophysiol 38:1403-1420.

Sprague JM, Levy J, DiBerardino A, Berlucchi G (1977) Visual cortical areas mediating form discrimination in the cat. $J$ Comp Neurol 172: $441-488$.

Sprague JM, Berlucchi G, Antonini A (1985) Immediate postoperative retention of visual discriminations following sclcctive cortical lesions in the cat. Behav Brain Res 17:145-162.

Sprague JM, De Weerd P, Vandenbussche E, Orban GA (1993) Orientation discrimination in the cat and its cortical loci. In: Progress in brain research, Vol 95 (Hicks TP, Molotchnikoff S, Ono T, eds), pp 381-400. Amsterdam: Elsevier.

Tanaka K, Ohzawa I, Ramoa AS, Freeman RD (1987) Receptive field properties of cells in area 19 of the cat. Exp Brain Res 65:549-558.

Toyama K, Fujii K, Umetani K (1990) Functional differentiation between the anterior and posterior Clare-Bishop cortex of the cat. Exp Brain Res 81:221-233.

Treisman A, Gelade $G$ (1980) A feature integration theory of attention. Cognit Psychol 12:97-136.

Tusa RJ, Palmer LA, Rosenquist AC (1978) The retinotopic organization of area 17 (striate cortex) in the cat. J Comp Neurol 177: 213-236.

Tusa RJ, Rosenquist AC, Palmer LA (1979) Retinotopic organization of areas 18 and 19 in the cat. J Comp Neurol 185:657-678.

Tusa RJ, Palmer LA, Rosenquist AC (1981) Multiple cortical visual areas. Visual field topography in the cat. In: Sensory organization (Woolsey CN, ed), pp 1-31. Clifton, NJ: Humana.

Uhlrich DJ, Essock EA, Lehmkuhle S (1981) Cross-species correspondence of spatial contrast sensitivity functions. Behav Brain Res 2:291-299.

Ungerleider LG, Mishkin M (1982) Two cortical visual systems. In: Analysis of visual behavior (Ingle DJ, Goodale MA, Mansfield RJW, eds), pp 549-586. Cambridge: MIT.

Vandenbussche E, Sprague JM, De Weerd P, Orban GA (1989) Effect of higher cortical ablations on cat orientation discrimination. Soc Neurosci Abstr 15:1255.

Vandenbussche E, Sprague JM, De Weerd P, Orban GA (1991) Orientation discrimination in the cat: its cortical locus. I. Areas 17 and 18. J Comp Neurol 305:632-658.

Vandenbussche E, Sprague JM, De Weerd P, Orban GA (1993) Texture segregation in the cat: interaction between orientation and contrast. Invest Ophthalmol Vis Sci 34:1237.

Van Essen DC, Maunsell JHR (1980) Two-dimensional maps of the cerebral cortex. J Comp Neurol 191:255-281.

Voorhees H, Poggio T (1988) Computing texture boundaries from images. Nature 333:364-367.

Wetherill GB, Levitt R (1965) Sequential estimation of points on a psychometrical function. Br J Math Stat Psychol 18:1-10.

Wilkinson F (1986) Visual texture segmentation in cats. Behav Brain Res 19:71-82.

Wilkinson F (1990) Texture segmentation. In: Comparative perception, Vol II, Complex signals (Stebbins WC, Berkley MA, eds), pp 125-156. New York: Wiley.

Wood CC, Spear PD, Braun JJ (1974) Effects of sequential lesions of suprasylvian gyri and visual cortex on pattern discrimination in the cat. Brain Res 66:443-466.

Yin TCT, Greenwood M (1992a) Visual response properties of neurons in the middle and lateral suprasylvian cortices of the behaving cat. Exp Brain Res 88:1-14

Yin TCT, Greenwood M (1992h) Visuomotor interactions in responses of neurons in the middle and lateral suprasylvian cortices of the behaving cat. Exp Brain Res 88:15-32. 